\title{
On-orbit performance of the Spitzer Space Telescope: science meets engineering
}

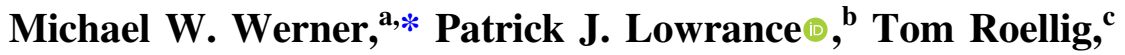 \\ Varoujan Gorjian $\odot,{ }^{\text {a }}$ Joseph Hunt, ${ }^{\text {a }}$ Charles M. Bradford, ${ }^{a}$ and \\ Jessica Krick ${ }^{b}$ \\ aJet Propulsion Laboratory, California Institute of Technology, Pasadena, California, \\ United States \\ ${ }^{\mathrm{b}}$ California Institute of Technology, IPAC-Spitzer, MC 314-6, Pasadena, California, \\ United States \\ ${ }^{c}$ NASA Ames Research Center, Space Sciences Division, Moffett Field, California, \\ United States
}

\begin{abstract}
The Spitzer Space Telescope operated for over 16 years in an Earth-trailing solar orbit, returning not only a wealth of scientific data but also, as a by-product, spacecraft and instrument engineering data that will be of interest to future mission planners. These data will be particularly useful because Spitzer operated in an environment essentially identical to that at the L2 LaGrange point, where many future astrophysics missions will operate. In particular, the radiative cooling demonstrated by Spitzer has been adopted by other infrared space missions, from JWST to SPHEREx. We aim to facilitate the utility of the Spitzer engineering data by collecting the more unique and potentially useful portions into a single, readily accessible publication. We avoid discussion of less unique systems, such as the telecom, flight software, and electronics systems, and do not address the innovations in mission and science operations that the Spitzer team initiated. These and other items of potential interest are addressed in references supplied in an appendix to this paper. () The Authors. Published by SPIE under a Creative Commons Attribution 4.0 International License. Distribution or reproduction of this work in whole or in part requires full attribution of the original publication, including its DOI. [DOI: 10.1117/1.JATIS.8.1.014002]
\end{abstract}

Keywords: infrared; space telescope; cryogenic; lessons learned.

Paper 21072 received Jul. 13, 2021; accepted for publication Dec. 22, 2021; published online Jan. 21, 2022.

\section{Introduction}

The Spitzer Space Telescope, a long-lived observatory operating outside the thermal and ionizing radiation environment of Earth orbit that makes use of both modern integrating infrared arrays and radiative cooling, serves as a technical pathfinder for the James Webb Space Telescope (JWST) and other future astrophysics missions. Rather than describe the entire Spitzer system design and performance in detail as found in Refs. 1 and 2, we concentrate our discussion of the on-orbit performance in areas in which we feel that the Spitzer experience most uniquely pertains to future missions. These are detailed in the following sections.

\subsection{Description of the Spitzer Space Telescope}

NASA launched Spitzer into an Earth-trailing solar orbit in August 2003 as a cryogenic telescope cooled by liquid helium and radiation to space. Table 1 summarizes for the readers the key properties of the Spitzer mission, and Fig. 1 shows a cutaway view of the observatory as flown. In considering these properties and the material below, readers should bear in mind that Spitzer was highly constrained in the 1990s, both in mass/volume by the required Delta launch vehicle and by NASA mandate to keep the cost through launch under $\$ 500 \mathrm{M}$ (it eventually grew to about $\$ 750 \mathrm{M}$, including launch vehicle). These constraints dovetailed nicely with the characteristics of

*Address all correspondence to Michael W. Werner, michael.w.werner@jpl.nasa.gov 
Werner et al.: On-orbit performance of the Spitzer Space Telescope: science meets engineering

Table 1 Top-level observatory parameters for the cryogenic mission. ${ }^{1}$

\begin{tabular}{lc}
\hline \hline Parameter & Value \\
\hline Total observatory mass at launch & $861 \mathrm{~kg}$ \\
Dimensions (height $\times$ diameter) & $4.5 \times 2.1 \mathrm{~m}^{2}$ \\
Average operating power & $375 \mathrm{~W}$ \\
Solar array generating capacity at launch & $500 \mathrm{~W}$ \\
Nitrogen reaction control gas at launch & $15.59 \mathrm{~kg}$ \\
Estimated reaction control gas lifetime & 17 years \\
Mass memory capacity & 2 Gbytes \\
Telescope primary diameter & $0.85 \mathrm{~m}$ \\
Telescope operating temperature (depending on instrument in use) & 5.6 to $13 \mathrm{~K}$ \\
Superfluid helium at launch & $337 \mathrm{~L}$ \\
Estimated nominal cryogenic lifetime & 5.6 to 6.0 years \\
As-commanded pointing accuracy (1 $\sigma$ radial) & $<0.5^{\prime \prime}$ \\
Pointing stability (1 $\sigma, 600 \mathrm{~s}$ ) & $\leq 0.03^{\prime \prime}$ \\
Maximum tracking rate & $1.0^{\prime \prime} \mathrm{s}^{-1}$ \\
Time to slew over $\sim 90$ deg & $\sim 8 \mathrm{~min}^{\prime}$ \\
Data transmission rate (high-gain antenna up to 0.58 AU from the Earth) & 2.2 megabytes s \\
\hline \hline
\end{tabular}

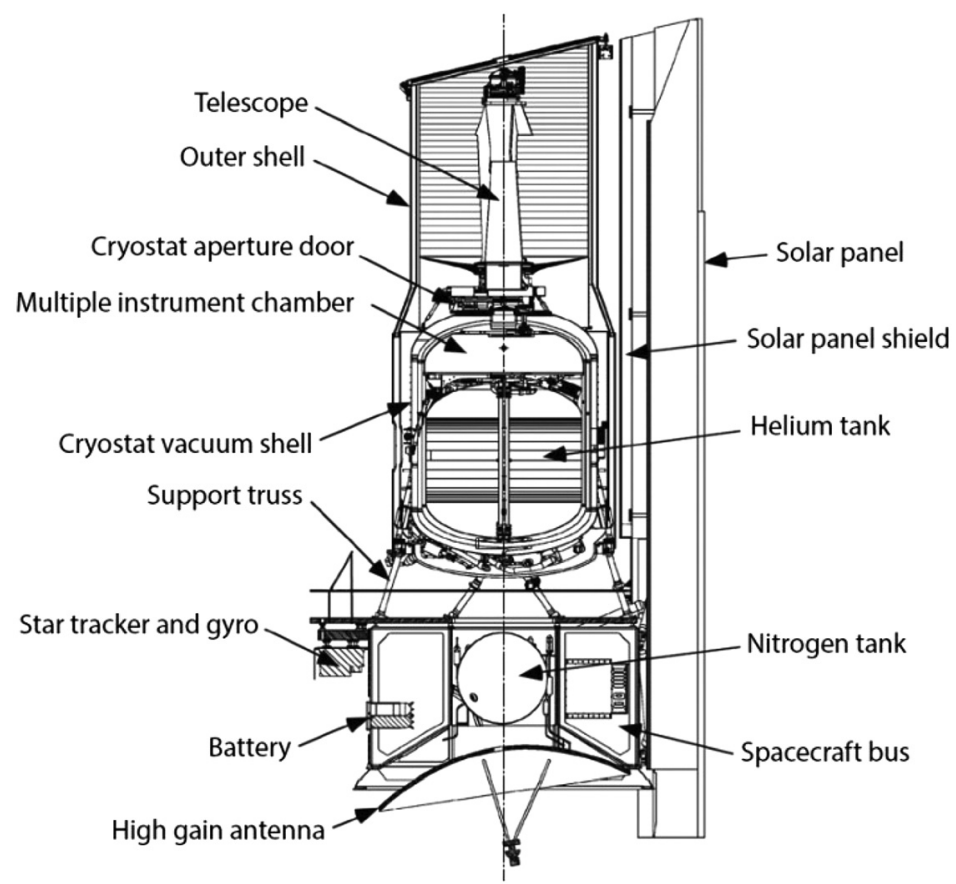

Fig. 1 Cutaway view of the Spitzer observatory. ${ }^{2}$ The dust cover atop the telescope tube was jettisoned a few days after launch, and the cryostat aperture door was opened shortly afterward to admit infrared radiation into the instrument chamber. See papers listed in the Appendix for a detailed discussion of the in-orbit checkout for Spitzer. 
the warm-launch, solar-orbiting mission that emerged as the optimum design solution, providing the most science per dollar. As exemplified by the discussion below of the warm mission, these cost constraints meant that not all conceivable analyses and design trades could be carried out.

\subsection{Cryogenic and Warm Missions}

Following depletion of the initial cryogen load in 2009, the Spitzer telescope and instruments warmed up to the point at which only the two shortest wavelength imaging channels remained operational. Spitzer operated in this warm mission mode very successfully until the mission was retired in early 2020. The development team realized from the first that the cryothermal architecture, with its heavy reliance on radiative cooling and the use of the telescope outer shell as a thermal boundary, would probably support the warm mission. However, it is important to note that NO resources were expended during the Spitzer development to optimize or prepare the system for the warm mission. Discussing the warm mission would inevitably distract the design/development teams, who were fully occupied with completing the cryogenic mission. In addition, the cost constraints did not make room for significant work on the warm mission. In NASA jargon, the cryogenic mission was the prime mission, and the warm mission was the extended mission. The Spitzer experience clearly shows that the best guarantee of a successful extended mission is careful work on the design and development of the prime mission.

\subsection{Spitzer's Orbit}

Unlike an observatory in Earth orbit or at L2, Spitzer had to address a separate set of challenges arising from the fact that, in the Earth-trailing solar orbit, the spacecraft, launched August 25, 2003, drifted away from the Earth at about 0.1 AU per year, reaching a distance of $1.75 \mathrm{AU}$ at the time of the end of the mission in January 2020. These challenges, discussed in detail in Ref. 3, arose from several considerations. The first was the reduced data downlink rate-from $2.2 \mathrm{Mbps}$ early in the mission to $0.55 \mathrm{Mbps}$ at the end-which was a consequence of the increased distance of Spitzer from Earth. This was compounded by the fact that Spitzer's fixed solar panel was optimized for solar incidence angles no greater than $30 \mathrm{deg}$. During the cryogenic mission, the incidence angle was always below $30 \mathrm{deg}$, and during the warm mission, the incidence angle was always kept below 30 deg while Spitzer was observing. However, because Spitzer downlinked through an antenna fixed to the bottom of the spacecraft, the communication geometry required larger solar incidence angles during the warm mission, reaching $55 \mathrm{deg}$ at the end of the mission. This had implications for battery utilization and recharging and led to solar illumination of structures at the lower end of the spacecraft not originally planned to be in direct sunlight. In addition, elements of the fault protection system had to be overridden to allow for the use of such large off sun angles without tripping fault protection limits. This had to be done as part of the setup for each download; following the download, the system was restored to its original configuration, so the fault protection would still be operational. Fortunately, the spacecraft engineering team at Lockheed-Martin, working with the mission operations team at the Jet Propulsion Laboratory and the science planners at the Spitzer Science Center, found a solution that met all of these constraints, at the cost of some operational flexibility as only a limited number of data-taking modes were allowed. Thus, Spitzer was able to operate with efficiency $~ 90 \%$ up to the end of its mission in January 2020. Efficiency is defined as (time spent on science, calibration, and slews)/ (wall clock time). We will not address these issues in greater detail. We anticipate that the much larger detector arrays anticipated for future missions, as exemplified by JWST, Euclid, and Roman, will inevitably point future missions to L2, where none of these problems need arise and where the thermal and sky-visibility benefits of the solar orbit remain. We did consider L2 instead of the solar orbit for Spitzer but felt that the mass, cost, complexity, and risk (to our cold surfaces) of the station-keeping made it less attractive than the solar orbit for the heavily constrained Spitzer.

It is instructive with regard to the previous discussion to note that Spitzer's orbit had an eccentricity of 0.0113 and a period of 373.1 days, slightly longer than an Earth year. Spitzer was inserted directly into this orbit by a final rocket burn, which ejected it from Earth orbit. It was this difference to the Earth's orbit that caused Spitzer to fall further behind the Earth

J. Astron. Telesc. Instrum. Syst. $\quad$ 014002-3 Jan-Mar 2022 • Vol. 8(1) 
every year. The Spitzer orbit was chosen both to assure that the observatory was not in danger of falling back to Earth and, more importantly, to allow Spitzer to escape rapidly from the Earth's heat load so that the radiative cooling could proceed. No station keeping was required in this orbit, and the cold $\mathrm{N}_{2}$ gas system described below was used only for momentum management, not for orbit maintenance. Finally, we point out that Spitzer was a robust and reliable spacecraft. Over the 16+ year mission, Spitzer averaged only slightly more than 1 safing or standby event per year. Fewer than 4 days/year were lost to these events.

\section{Thermal Considerations}

\subsection{Thermal System Design and Performance: Use of Radiative Cooling}

The discussion below will refer repeatedly to the heat flow diagram for the cryogenic mission shown in Fig. 2. Paul Finley at Ball Aerospace developed this model, which represents a correlation of the on-orbit performance of the thermal system early in the cryogenic mission with the prelaunch thermal model. Unfortunately, no comparable heat flow diagram exists for the warm mission, but some of the elements of Fig. 2 bear directly on the thermal performance of the warm mission.

Spitzer was launched with its cryostat containing 337 l (49 kg) of superfluid liquid helium, which cooled the instruments and the telescope. Spitzer operated in this cryogenic mission mode until mid-2009, utilizing all three science instruments and observing at wavelengths between 3 and $180 \mu \mathrm{m}$. When the helium supply was exhausted in mid-May of 2009, the system warmed up to a level at which only the two shortest wavelength arrays of the Infrared Array Camera

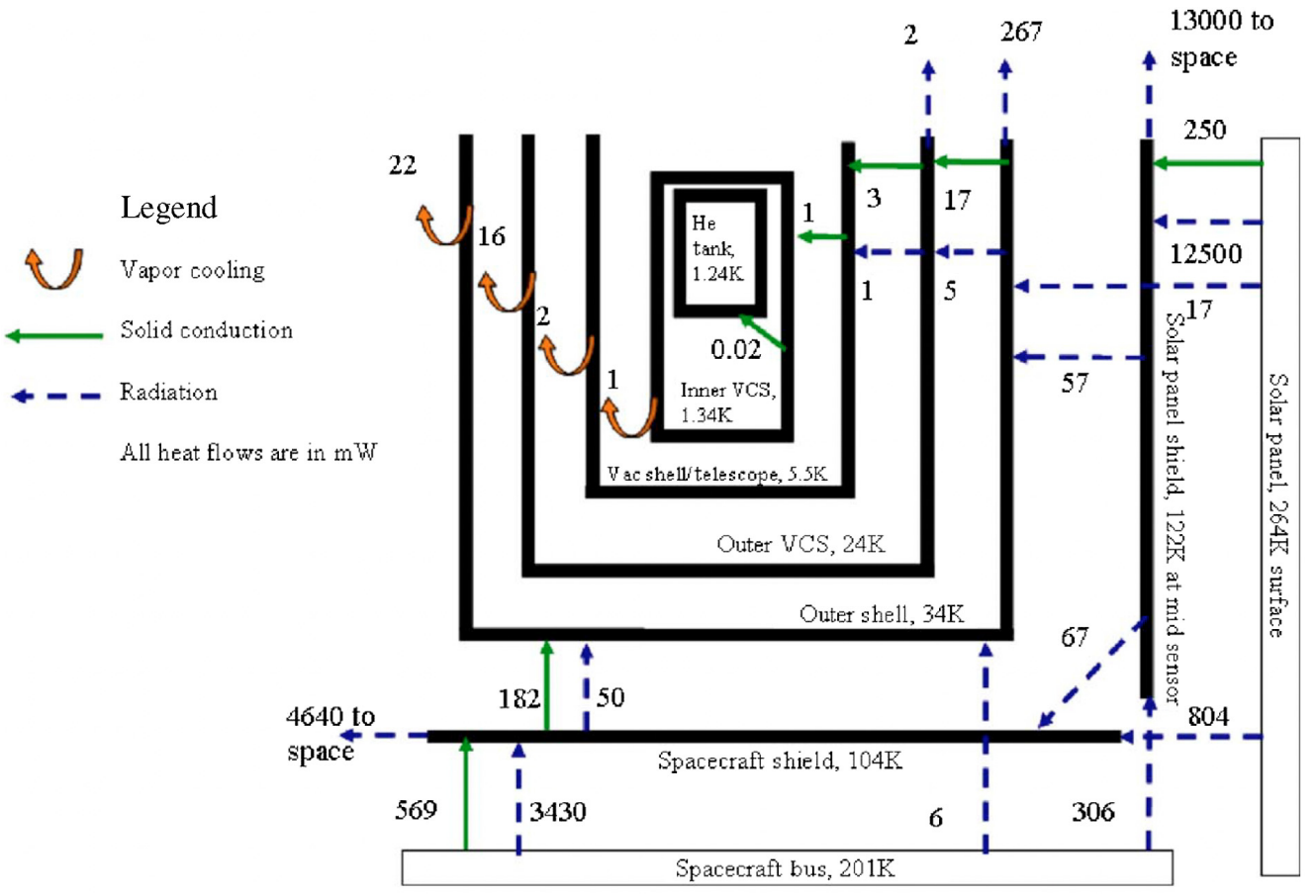

Heat flow map

Flight-correlated model

Fig. 2 Ball Aerospace thermal model. ${ }^{1}$ Heat input is solely from insolation on the solar panel. Cooling of the cryogenic telescope assembly is accomplished by radiation and vapor cooling. Heat is transferred through the system along the paths indicated by the arrows by radiation (dashed blue arrows), conduction (solid green arrows), and vapor cooling (broad orange arrows). The equilibrium temperatures for the various observatory components are given for the case when the cryogenic telescope is operating at $5.5 \mathrm{~K}$. The model assumes a focal-plane heat dissipation of $4 \mathrm{~mW}$ and an insolation of $5.3 \mathrm{~kW}$. Courtesy of Ball Aerospace/JPL-Caltech. 
(IRAC) instrument, operating at 3.6 and $4.5 \mu \mathrm{m}$, had low enough dark current to be useful for scientific observations. This phase, referred to below as the warm mission, lasted until the observatory was turned off in January 2020 . Here we discuss the thermal history of key components of Spitzer's thermal system over the entire 16+ year mission. Figures 1 and 2 illuminate this and the following discussions. Note that, unlike the previous Infrared Astronomical Satellite (IRAS) and Infrared Space Observatory (ISO) missions, which launched with the telescope inside the cryostat, the Spitzer telescope was launched warm and cooled on orbit, making maximum use of radiative cooling to lose energy to the coldness of space.

Some idea of the overall efficiency of the Spitzer cryogenic system is given by the following. After the loss of helium due to blowdown and on-orbit cooling, the observatory entered its final stages of in-orbit checkout on October 10, 2003, carrying $43.4 \mathrm{~kg}$ of liquid helium, down from the $49 \mathrm{~kg}$ present at launch. This lasted through May 15, 2009, a total of 2030 days, so the helium was boiled away at a rate of $0.24 \mathrm{mg} / \mathrm{s}$. The corresponding average heat load to the helium bath was $5.1 \mathrm{~mW}$, consistent with the power dissipated at the focal plane by the instruments and make-up heaters. In comparison, IRAS carried $73 \mathrm{~kg}$ of liquid helium and had a lifetime, in low Earth orbit, of 10 months, as opposed to the over 5.5 years achieved by Spitzer with $42.5 \mathrm{~kg}$ of liquid helium. IRAS's helium usage was dominated by parasitic heat conducted and radiated inward from the warm outer shell of the cryostat; Spitzer, using radiative cooling in the thermally advantageous heliocentric orbit to cool the telescope outer shell, had helium utilization dominated by the much lower power level needed to operate the arrays and the make-up heater used to control the helium bath temperature and thus the telescope temperature as well (see Sec. 2.5). Although improvements to the Spitzer thermal design could be contemplated, we emphasize that the heat load to the helium bath was dominated by the unavoidable (though minimal) power demands of the focal plane instruments. Thus, the cryogenic lifetime was in fact determined by the amount of helium remaining after launch and initial cooldown. The size of the cryostat was limited by the constraints discussed above, so the only design improvements that could significantly increase the cryogenic lifetime would be ones that minimized the amount of helium used in the initial mission phases, and none have been suggested.

\subsection{Solar Panel}

We discuss the thermal history of key elements of the Spitzer thermal/cryogenic system, starting at the outside and working inward. The photograph in Fig. 3 shows the elements of the thermal system external to the telescope. Spitzer was optimized for studies at infrared wavelengths, which required that the telescope, its baffles, and the instruments be cooled to below $10 \mathrm{~K}$. To achieve this within the cost and mass constraints imposed programmatically, the telescope was designed and operated to make maximum use of radiative cooling as shown in Fig. 2. During the cryogenic mission, the fixed solar panel (cf. Fig. 3) was always oriented to shade the telescope outer shell and the spacecraft from the sun, and the antisolar side of the outer shell was painted black to enhance its radiative cooling power. The solar panel assembly was provided by Lockheed-Martin as part of the spacecraft. Most of the rest of the flight system, including the solar panel shield, the spacecraft shield, the outer shell, and the structures within it, as well as the Infrared Spectrograph (IRS) and Multiband Imaging Photometer (MIPS) instruments, was provided by Ball Aerospace. Goddard Space Flight Center provided the third instrument, the Infrared Array Camera (IRAC), which was the only instrument in use during the warm mission and features prominently in this paper.

To understand the thermal performance of Spitzer, we start at the solar panel, which absorbs and redistributes all of the power-in the form of both electrical and thermal energy - coursing through the Spitzer system. The solar panel was divided into two sections. The lower portion, consisting of two $330 \times 72 \mathrm{~cm}$ panels arranged in a chevron arrangement (Fig. 3), was 60\% covered with $11 \mathrm{mil} \mathrm{GaAs} / \mathrm{Ge}$ solar cells with $18 \%$ efficiency at launch, similar to those that were flown on the Iridium spacecraft. The solar cells provided more than the $\sim 375 \mathrm{~W}$ average operating power required by Spitzer throughout the mission. An aluminum upper section extended the chevron an additional $\sim 160 \mathrm{~cm}$ above the active section of the solar panel and shaded the upper section of the telescope outer shell from direct sunlight. Thus, the solar panel served as both sunshade and electric power generator. The sun-facing side of this extension and

J. Astron. Telesc. Instrum. Syst. $\quad 014002-5 \quad$ Jan-Mar 2022 • Vol. 8(1) 


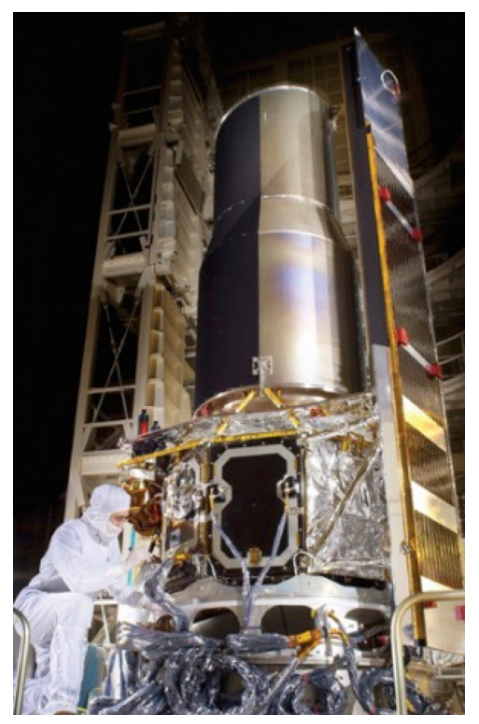

Fig. 3 The assembled Spitzer observatory being prepared for thermal vacuum testing at Lockheed Martin in Sunnyvale, California. The photograph illustrates the key features of the thermal control system up to and including the outer shell of the telescope. These include the chevronshaped solar panel, the solar panel shield immediately behind it, and the telescope outer shell, with its antisolar side painted black to maximize its infrared emissivity. Also visible between the spacecraft and the outershell is the spacecraft shield, which isolated the outer shell from the spacecraft. Credit: NASA.

the regions of the lower section not occupied by solar cells were covered with second-surface aluminized Teflon with an absorption/emissivity ratio of $0.26 / 0.80$, designed to minimize the temperature of the solar panel.

Figure 4 shows the average temperature of the active lower segment of the solar panel as a function of time throughout the Spitzer mission. The temperature shows a gradual increase in time, superposed on a periodic annual variation due to the eccentricity of Spitzer's orbit. Over the $16+$ year mission, the temperature of the active portion of the solar panel increased from 338 to $344 \mathrm{~K}$, corresponding to an increase in absorbed power of $(344 / 338)^{4}-1=0.073$, or $7.3 \%$, because the solar panel cools principally by radiation. This increase is attributed by Ref. 4 to "unsurprising degradations in thermal coatings on the solar panel." Similar increases in temperature are seen in other long-lived space systems, and are attributed, as suggested above, to degradation of the solar panel materials by the effects of unfiltered solar ultraviolet light and the energetic particle environment in space. In the case of Spitzer, the increased solar panel temperature is accompanied by a drop in the electrical output (discussed below), which may also be attributed to degradation of the optical properties of the solar panel materials.

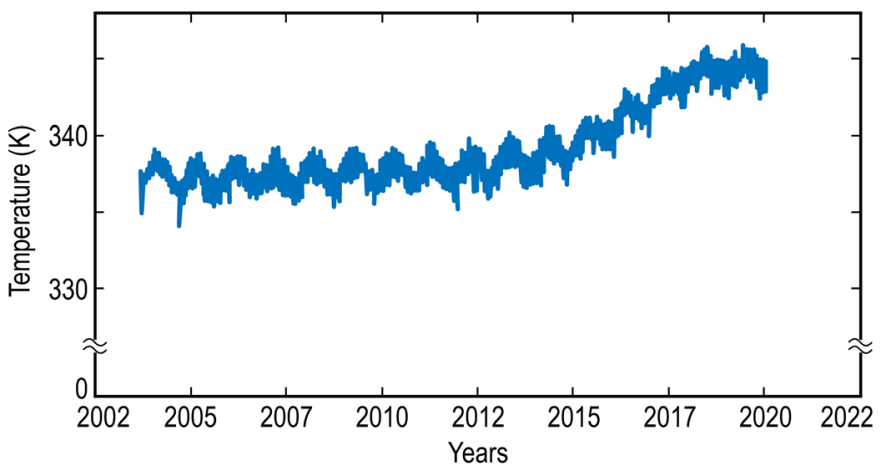

Fig. 4 The average temperature of the lower, solar cell-containing portion of the solar panel versus time over the entire Spitzer mission. The annual periodicity seen in this and the other temperature data reflects the eccentricity of Spitzer's orbit. 


\subsection{Solar Panel Shield and the Outer Shell}

The solar panel shield lies between the solar panel and the outer shell described below (see Fig. 3). Structurally, the solar panel shield and the outer shell are constructed of lightweighted aluminum honeycomb cores with thin face sheets. The solar panel shield and the solar panel are stood off from the spacecraft by Gr/CE struts and not physically attached to any portion of the telescope outer shell. The surface finishes are chosen to control heat flow-low emissivity surfaces, such as the facing sides of the outer shell and the solar panel shield, are coated with cocured aluminized Kapton. By contrast, as shown in Fig. 3, the space facing back half-cylinder of the outer shell is coated with a proprietary Ball black paint, ${ }^{5}$ which gives it very high emissivity even at the low temperatures achieved on orbit. As shown in Fig. 2, the solar panel shield coupled to the solar panel primarily through radiation, with only $2 \%$ of the heat transferred by conduction. The solar panel shield loses most of this energy by radiation to space, thereby greatly reducing the heat load on the outer shell. Multilayer insulation thermal blankets were used to reduce the temperatures of the solar panel shield and the spacecraft shield (Fig. 3), which were adjacent to the telescope outer shell, to 122 and $104 \mathrm{~K}$, respectively. One blanket lay between the solar panel and the solar panel shield, and the other was positioned below the spacecraft shield.

All of the energy that found its way into the critical telescope/cryostat structure passed through the outer shell. Thus, the telescope outer shell was a critical node in the thermal system as it set a thermal boundary for the telescope and the cryostat, which sit within it. The cryostat contained the liquid helium tank and the instrument chamber, which is thermally coupled to the helium tank and not to the cryostat vacuum shell. On orbit, the heat input to the telescope outer shell, a structure $\sim 3 \mathrm{~m}$ in length and $\sim 1.2 \mathrm{~m}$ in diameter, was about $310 \mathrm{~mW}$, which is one part in 17,000 of the $5.3 \mathrm{~kW}$ striking the solar panel. The outer shell was heated approximately equally by radiation and conduction (Fig. 2). The radiation was from the solar panel shield and from the spacecraft shield, which lay between the spacecraft and the outer shell. The conduction was from the mechanical and electrical connections between the spacecraft and the outer shell; the microcables passing inward to the cryostat and the instruments were heat sunk to the outer shell via its supporting truss of low conductivity gamma-alumina struts. The outer shell lost energy through radiation to space and, to a much lesser extent, by conduction inward. Because the radiative and conductive heat loads into the outer shell are comparable, the system was "well-balanced" in a thermal sense. During the cryogenic mission, the heat conducted inward was carried away by the last stages of vapor cooling provided by the evaporated helium as it left the system through a low-thrust valve. It is important to note that the heat loads to the outer shell did not vary dramatically between the cryogenic and warm missions beyond the gradual increase implied by the thermal histories shown in this paper.

As the power absorbed by the solar panel increased over the mission, a corresponding rise occurred in the temperature of the outer shell. Figure 5 shows the outer shell temperature as a

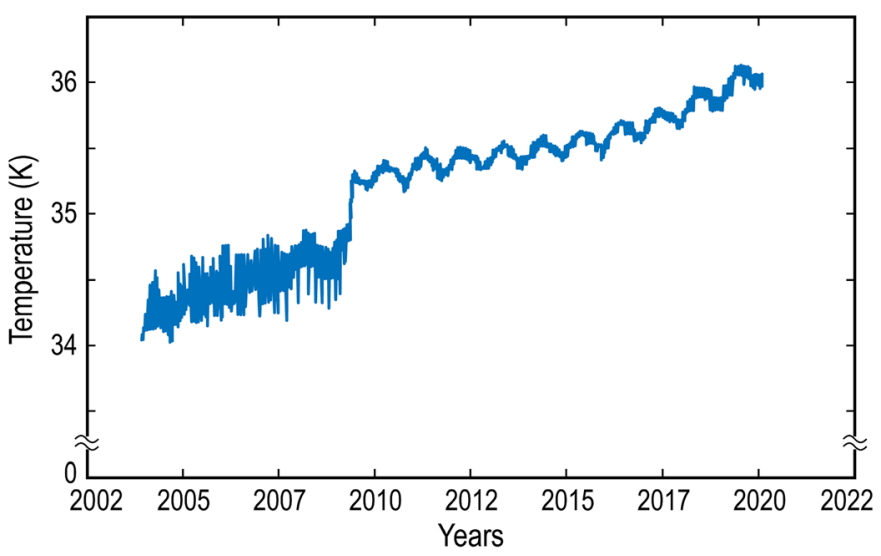

Fig. 5 Spitzer outer shell temperature versus time over the entire Spitzer mission. The discontinuity in 2009 reflects the exhaustion of the liquid helium and the start of the warm mission, when vapor cooling was no longer available. The oscillations in temperature seen before this reflects the variation in vapor cooling resulting from the helium utilization strategy described in the text. 
function of time over the entire 16+ year Spitzer mission. The step upward rise in temperature by about $0.5 \mathrm{~K}$ seen in 2009 marks the exhaustion of the liquid helium and the start of the warm Spitzer mission. At this point, the absence of vapor cooling allowed the outer shell to warm slightly. It also allowed more heat to be conducted inward to the structures within the outer shell. These structures warmed far above the temperatures characteristic of the cryogenic mission. The outer shell temperature continued to rise slowly after this, as expected from the rising temperature of the solar panel, which let more heat into the Spitzer system. We also see the annual variation of temperature due to the eccentricity of Spitzer's orbit superposed on this long-term trend. The relatively large fluctuations in temperature prior to helium exhaustion in mid-2009 reflected fluctuations in the amount of helium gas evaporating in the cryostat and hence in the cooling power of the escaping helium vapor and, again, in the temperatures of the structures interior to the outer shell. These periodic fluctuations result from the helium utilization strategy discussed below. Starting after helium depletion in mid-2009, however, this cooling path was no longer in effect, but the design and operational features that enabled radiative cooling during the cryogenic mission were still in place. These features kept the outer shell temperature below $36 \mathrm{~K}$ at the start of the warm mission, maintained entirely passively.

Note that the envelope of the temperature fluctuations prior to mid-2009 shows that the temperature rise began immediately after launch. ${ }^{4}$ The increase in outer shell temperature mirrored the increase in solar panel temperature. The data in Fig. 5 show an increase in temperature from $\sim 35.3$ to $\sim 36 \mathrm{~K}$ during the warm mission. To isolate the performance of the radiative cooling system external to the outer shell, we extrapolate the data back to the start of the mission and remove the offset due to the loss of vapor cooling. The envelope of the curve suggests that perhaps another $0.4 \mathrm{deg}$ increase in temperature would have been recorded. Thus, the power radiated by the outer shell in this slightly fictional scenario increased by $(36 / 34.9)^{4}-1=13 \%$. Absent a complete thermal model, this can be taken as an upper limit for the degradation in the performance of the radiative cooling alone because some of the increase must be simply in response to the increased temperature of the solar panel. Note that the outer shell loses energy predominantly by radiation. In reality, of course, the power radiated by the outer shell increases by closer to $20 \%$ when the effects of the loss of the vapor cooling are taken into account. Because of the factor of 10 difference in the temperatures of the two systems, this $20 \%$ increase in the power radiated by the cold outer shell is not inconsistent with the $7 \%$ increase in power radiated by the much warmer solar panel.

\subsection{Telescope}

The telescope optics and metering structure were fabricated of hot isostatically pressed beryllium. Beryllium was chosen because of its favorable strength to weight ratio and its mechanical stability at low temperature. The optics were polished but not coated. The telescope and its barrel baffle lay within the outer shell and were surrounded by a vapor cooled shield that intercepted energy conducted and radiated inward from the outer shell. The telescope was thermally anchored to the exterior vacuum shell of the cryostat, which, in turn, was cooled by the helium boiloff. Two vapor cooled shields provided additional thermal isolation of the helium tank and its associated instrument chamber from the cryostat vacuum shell. The instrument chamber contained the three instruments and the Pointing Calibration and Reference Sensor (PCRS), discussed further below. As the helium boil-off rate was varied according to the strategy described below, the cryostat outer shell and the telescope temperatures varied to meet the needs of the instrument in use. This scenario accounted for both the low temperature of the primary mirror and the temperature fluctuations seen during the cryogenic mission prior to mid-2009 (Fig. 6). Except for the detectors, the instrument hardware was thermally anchored to the helium tank and would rise and fall in temperature very slightly as the bath temperature changed. During the cryogenic mission, the InSb and Si:As IBC detectors used below $40 \mu \mathrm{m}$ were thermally stabilized at temperatures above the enclosure temperature, whereas the Ge:Ga photoconductors in the MIPS instrument, which had to operate below $2 \mathrm{~K}$, were thermally strapped directly to the helium bath.

During the cryogenic mission, the instrument temperature varied slightly due to the helium utilization scheme described below. No effects on the instrument optical performance were seen

J. Astron. Telesc. Instrum. Syst. $\quad$ 014002-8 Jan-Mar 2022 • Vol. 8(1) 


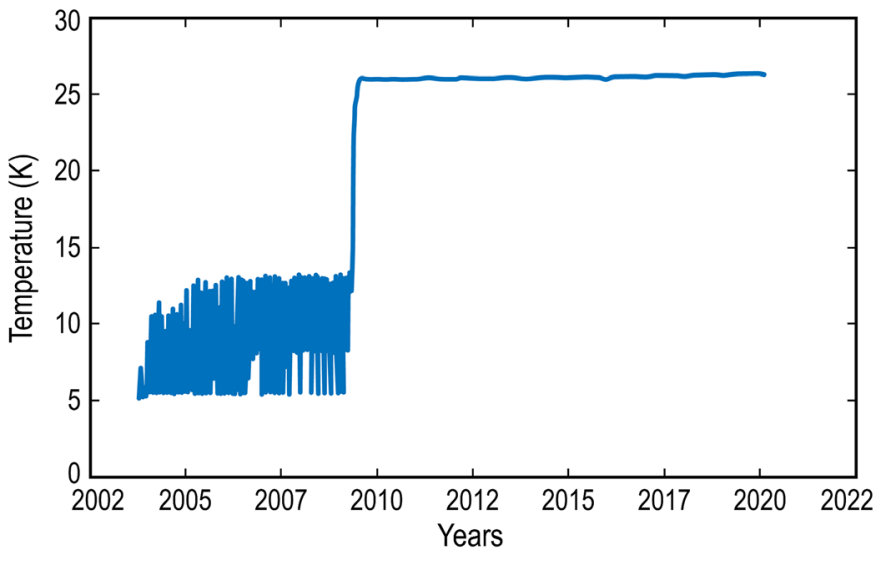

Fig. 6 Spitzer primary mirror temperature versus time over the entire Spitzer mission. The fluctuations in temperature seen prior to the start of the warm mission in mid-2009 reflect the helium utilization strategy described in the text.

as a result of these changes. More tellingly, because of the thermal stability of beryllium, it was neither planned nor necessary to refocus the telescope following the transition to the warm mission, during which the telescope temperature shifted upward from $\sim 10 \mathrm{~K}$ to $\sim 25 \mathrm{~K} .{ }^{6}$ Over a larger temperature change, it was noted during IOC that the position of the telescope focus stabilized to within $0.01 \mathrm{~mm}$ once the telescope temperature fell below $\sim 50 \mathrm{~K}$.

During the warm mission, the heat load applied within the cryostat, a combination of power required to stabilize the two IRAC arrays in use and the power dissipated in reading the arrays out, was constant with time at a level of $1.4 \mathrm{~mW}$. The effects of the increasing outer shell temperature and its annual variation are just visible in the telescope temperature, which was stable at 26 to $27 \mathrm{~K}$ throughout the warm mission (Fig. 6). The tight mechanical coupling of the telescope, the cryostat, and the barrel baffle suggest that all structures within the vapor cooled shield were at about the same temperature, and these interior structures remained at $\sim 26 \mathrm{~K}$ by radiating about $20 \mathrm{~mW}$ into space through the open end of the outer shell. This suggests that, in addition to the $1.4 \mathrm{~mW}$ dissipated within the cryostat by the IRAC arrays, a bit more than an additional $20 \mathrm{~mW}$ was conducted or radiated from the outer shell through the shield and into these interior structures. Although a detailed thermal model for warm Spitzer does not exist, this lies in the range of the conductive and radiative loads into the interior of the outer shell seen in the heat flow diagram (Fig. 2). It is important to realize that these loads should be about the same in the warm mission, but they now warmed the telescope because the cooling effect of the evaporating helium gas was no longer present to counteract them.

\subsection{Maximizing the Cryogenic Lifetime of Spitzer}

Spitzer operated only one instrument at a time (typically for a 1-week campaign), as appropriate for a system in which the cryogen boil-off rate was set by the power dissipated by the instruments within the cryostat. If two instruments were in use simultaneously, the helium would have been used at a correspondingly higher rate, shortening the mission and reducing the time available for scientists to plan future observations based on initial results. This single-instrument mode enabled the following strategy for maximizing the cryogenic lifetime of Spitzer.

The fluctuations in the primary mirror temperature during the cryogenic mission (Fig. 6) reflect a tailoring of the mirror temperature to the needs of the instrument being used. Through the use of a heater that increased the rate at which helium was evaporated in the cryostat, the telescope was kept cold enough $(5.5 \mathrm{~K})$ for natural-background-limited operation when the long wavelength MIPS instrument was in use. The heater was turned off and the telescope temperature was allowed to drift upward when the shorter wavelength instruments-IRAC and IRS-took over. This tailoring increased the cryogenic lifetime of the system by at least 6 months over an approach in which the mirror temperature was always kept at $5.5 \mathrm{~K}$. 
It may have application to future missions that carry expendable cryogens and is described in detail by Ref. 7 and summarized by Ref. 8 .

\subsection{Integration, Test, and Verification of the Cryothermal System}

A prelaunch test of the performance of the cryothermal system was necessary to assure that Spitzer would meet its requirement of 2.5 years cryogenic lifetime (the prelaunch goal was 5 years). The NASA mantra "test as you fly" was, unfortunately, not applicable to tests of Spitzer's cryothermal system due to the impracticability of replicating the very low thermal background of space, together with solar insolation on one side, in any test chamber large enough to accommodate the entire Spitzer observatory. Instead, we broke this verification into two parts, with the boundary at the outer shell. The reasoning was simple: in flight, all heat input into the telescope, instruments, and cryogen system was routed through the outer shell; thus, the telescope temperature and the helium flow rate would depend only on the outer shell temperature and not directly on any external heat sources. The thermal performance of the structures within the outer shell was to be tested in the thermal balance test at Ball Aerospace, carried out with fixed outer shell temperatures.

The thermal performance of the hardware external to the outer shell, which established the outer shell temperature, was evaluated as part of the system level thermal vacuum test, which was carried out at much higher temperatures. In neither case did the test conditions faithfully replicate the on-orbit conditions, so much of the test effort was devoted to validating the models that were to be used to understand the test results and to extrapolate from the test to the orbital environment. These tests evaluated not only the design of the thermal system but also the quality of the workmanship involved in its assembly.

Reference 9 describes the low temperature thermal test and verification of the outer shell and its interior components. The tests were carried out in a chamber at Ball Aerospace with walls cooled by liquid nitrogen, with the telescope, the cryostat, and the instruments assembled for launch within the outer shell (the thermal test was done concurrently with an end-to-end optical test described below). The outer shell was shielded from a direct view of the chamber walls by extensive thermal shields and blankets. The original intent of the test was to set the outer shell temperature at both nominal and worst-case values expected on orbit and to measure the telescope temperature and helium flow rate in each case. The outer shell temperature was to be controlled by circulating cold helium vapor through a cooling loop at the base of the outer shell. This cooling loop was included in the design for just this purpose, illustrating the importance of outlining the test strategy during the design phase for a complex, difficult to test system such as Spitzer.

However, data taken during the initial cool down showed that the heat load on the vacuum shell of the cryostat, which lay interior to the outer shell and was mechanically and thermally coupled to the telescope, was 5 to 10 times the 5 to $10 \mathrm{~mW}$ expected on orbit. This uncontrolled power was small compared with the potential test induced heat input from radiation or solid or gaseous conduction (there were four separate cooling loops bringing in helium from vessels outside the test chamber); for example, a blackbody at $273 \mathrm{~K}$ radiates about $30 \mathrm{~mW} / \mathrm{cm}^{2}$. The challenge detailed in Ref. 9 was to separate the environmental or test-induced heat loads from those intrinsic to the system; only the latter would be present in flight. To do this, the authors did a careful audit of the possible test-induced heat loads, which are described in detail in Ref. 9, to determine which were credible and incorporated them into a thermal model of the test configuration, which also included the parameters characterizing the thermal performance of the flight system. They identified numerous possible sneak heat paths, including, for example, radiation down the pipes used in cooling loops that carried liquid helium from outside the test dewar into the outer shell. Following correction of these known problems, there were quite a few parameters to be evaluated, and the long thermal time constant of the system made it impractical to test each separately. Instead, a series of eight separate energy balance cases, each with a different set of environmental and system parameters, was carried out and analyzed in detail. Because the system never reached thermal equilibrium during these tests, much of the analysis focused on the transient behavior of the system, which was predicted by the thermal model. The transient behavior reflected the thermal coupling between various system elements, which was central to

J. Astron. Telesc. Instrum. Syst. 1014002-10 Jan-Mar 2022 • Vol. 8(1) 
the on-orbit performance. In short, the purpose of the telescope thermal balance test became validation of the thermal model rather than actual demonstration of flight performance.

This painstaking work succeeded in separating the test artifacts—which were still dominant - and in predicting on-orbit behavior. It set the stage for the observatory level thermal balance test of the assembled spacecraft, carried out in a large chamber at Lockheed Martin, Sunnyvale, and shown in Fig. 3. As was the case for the test of the outer shell and its interior systems, described above, it was not possible during the observatory thermal balance test (which had other objectives in addition to supporting an estimate of the cryogenic lifetime) to replicate the boundary conditions that the observatory would encounter in space. Instead, the analysis focused on three time windows, spread over about 3 days, during which temperatures of key system elements were measured as the observatory cooled down. Prominent among the elements investigated were the spacecraft shield and the solar panel shield because a key objective was to test whether these components, which were expected to dominate the radiative and conductive loads on the outer shell during flight, could adequately isolate the outer shell from the heat of the solar panel and the spacecraft. Of course, the outer shell temperature was monitored as well. In a similar spirit to that of the outer shell test described above, the analysis focused on the changes in temperature from one time window to another rather than on the actual temperatures, which were greatly influenced by environmentally induced heat loads.

Comparison of the actual flight performance with the prelaunch predictions based on the tests described above shows that the latter were remarkably accurate. The analysis ${ }^{10}$ of the prelaunch data, following the thermal balance test, showed that even the worst case (when all thermal parameters were stacked up with their least favorable values) yielded a cryogenic lifetime of 3.1 years, considerably longer than the 2.5 year requirement, whereas the nominal cryogenic lifetime was predicted to be 5.1 years. This lifetime analysis assumed that the telescope was constantly cooled to its lowest required operating temperature of $5.5 \mathrm{~K}$.

It was already apparent that the adaptive cryogenic utilization scheme described above would increase the lifetime by $5 \%$ to $10 \%$, leading to a more realistic lifetime prediction of 5.4 to 5.6 years. These prelaunch predictions agreed extremely well with the actual on-orbit cryogenic lifetime of just under 5.7 years. Similarly, the predicted temperatures ${ }^{9}$ of specific components during the cryogenic mission agreed very well with the on-orbit data. Most importantly, the outer shell temperature was predicted to be $32 \mathrm{~K}$, very close to the observed value of 34 to 34.5 K (Fig. 5).

Finally, when the thermal model was adjusted following launch to fit better the actual temperatures measured during the cryogenic mission, it predicted temperatures during the warm mission of $24 \mathrm{~K}$ for the telescope and $36 \mathrm{~K}$ for the outer shell (P. Finley, private communication). Again, these predictions were in good agreement with the measured values shown earlier (Figs. 4 and 5). It is noteworthy that almost 20 years ago the state-of-the-art thermal modeling led to very accurate predictions of the on orbit behavior of a complex system that utilized both radiative and cryogenic cooling. The performance and predictability of Spitzer's radiative cooling approach has been important in reducing the risk of using radiative cooling in other NASA missions, such as JWST and SPHEREx.

In summary, the performance of the Spitzer cryogenic system on orbit was remarkable. It is a tribute not only to the careful design of the system by our partners at Ball Aerospace but also to the extreme care with which it was assembled and tested. For lessons learned from this experience, we refer the readers to the publications by the Ball group ${ }^{4,9-11}$ as well as the more general lessons learned analysis of Ref. 12. Two lessons that stick out, however, are the following: (1) for a complex cryothermal test such as that undertaken for Spitzer, the design and fabrication of the test configuration and other GSE need as much care and attention as is given to design and fabrication of the flight system, and (2) it is important to remember that, for systems such as Spitzer for which the on-orbit thermal environment cannot be duplicated in the lab, the thermal balance tests can be used to validate the system thermal model, which can then be used with greater confidence to predict the on-orbit behavior. In this modeling and validation, it may be found that tests constructed and instrumented to permit analysis of the transient behavior of the system are equally valuable, and considerably easier to implement, than those that rely on reaching a steady state temperature, which may take a prohibitively long time. 


\section{Payload Issues}

\subsection{Optical System Verification and Focus Adjustment}

Spitzer used an all-beryllium $85-\mathrm{cm}$ diameter $f / 12$ Cassegrain telescope that illuminated a focal plane $\sim 30$ arcmin in diameter. The focal plane housed pickoff mirrors which fed the modules of all three Spitzer instruments as well as the PCRS. The telescope was body pointed to place the target of interest onto the pickoff mirror, and hence the entrance aperture, of the instrument/ module to be used for a particular observation. This design required that the instrument modules be confocal; this was achieved by designing and testing on the ground and was verified on-orbit. The telescope with the instruments installed underwent optical test and verification at cryogenic temperature in the large cryogenic test chamber at Ball Aerospace. These tests were simultaneous and interleaved with the thermal verification tests described above. A standard double-pass test using a cold flat mounted above the telescope and a short wavelength infrared source placed at the telescope focal plane by Ball for just this purpose, illuminated the IRAC arrays at 3.6 and $4.5 \mu \mathrm{m}$. The test validated the end-to-end image quality of the system, discussed further below. It also allowed the Spitzer team to use the focus mechanism, which was part of the secondary mirror assembly, to set the focus at the estimated position expected for the zero-gravity postlaunch configuration. The primary mirror was polished to have the right configuration for its cryogenic performance by measuring the distortions in the figure at cryogenic temperature and polishing the inverse of the measured deviations into the mirror at room temperature. Following the warm polishing of the mirror and an initial cryogenic test, one additional polishing cycle was carried out following this approach, after which a second cryogenic test confirmed that the mirror met specifications. Further details concerning the design, fabrication, test, and performance of the optical system are given by Ref. 1 .

The approach taken on orbit to determine and then set the optimum position for the secondary mirror may be relevant to future space observatories. The focus mechanism provided motion only along the optical axis and was robustly designed and electrically redundant. Nevertheless, there was an understandable reluctance to carry out a traditional focus sweep that would require many activations of the focus mechanism at cryogenic temperature. Instead, a group led by Bill Hoffmann from the University of Arizona developed a technique for determining focal position by looking at the variation of image quality across the combined $\sim 10$ arcmin field of view of the two short wavelength IRAC arrays at 3.6 and $4.5 \mu \mathrm{m}$. This technique, described by Ref. 13 and summarized by Ref. 8, was validated through a double-blind simulation on the ground ${ }^{14}$ and was successfully applied on orbit. A series of on-orbit measurements showed that the position of the telescope focus had stabilized to better than $0.01 \mathrm{~mm}$ once its temperature fell below $\sim 50 \mathrm{~K},{ }^{1}$ as was expected from the thermomechanical properties of beryllium and the other materials in the mechanical assembly. At this point, the analysis ${ }^{13}$ showed that the telescope focus lay $1.8 \mathrm{~mm}$ above the nominal focal plane established by the instrument entrance apertures. A small test move of the focus mechanism verified the direction of motion. Then, a larger motion of the secondary mirror brought the focus position within the required range. The telescope optics alone provided an image FWHM of $\sim 1.45 \operatorname{arcsec}^{15}$ at the center of the 3.6 and $4.5 \mu \mathrm{m}$ arrays. However, when the effects of the instrument optics and array pixelization are considered, the images in the reduced data have FWHM 1.6 to 2". ${ }^{16}$ This image quality has supported Spitzer studies of highly redshifted starlight from galaxies in the distant universe.

\subsection{Instrumental Sensitivity}

With its very cold optics and careful stray light baffling, the Spitzer observatory achieved photometric sensitivities close to the limits imposed by natural backgrounds in its broad band imaging instruments. Figure 7 shows a comparison of the measured Spitzer instrument sensitivity compared with the natural background limit due to the zodiacal emission in the direction of the North Ecliptic Pole. The calculated natural background limit includes the measured Spitzer instrumental optical throughput as detailed in the figure caption but assumes otherwise perfect instruments with noiseless detectors and $100 \%$ on-source observing efficiency. The natural background limit

J. Astron. Telesc. Instrum. Syst. $\quad$ 014002-12 Jan-Mar 2022 • Vol. 8(1) 


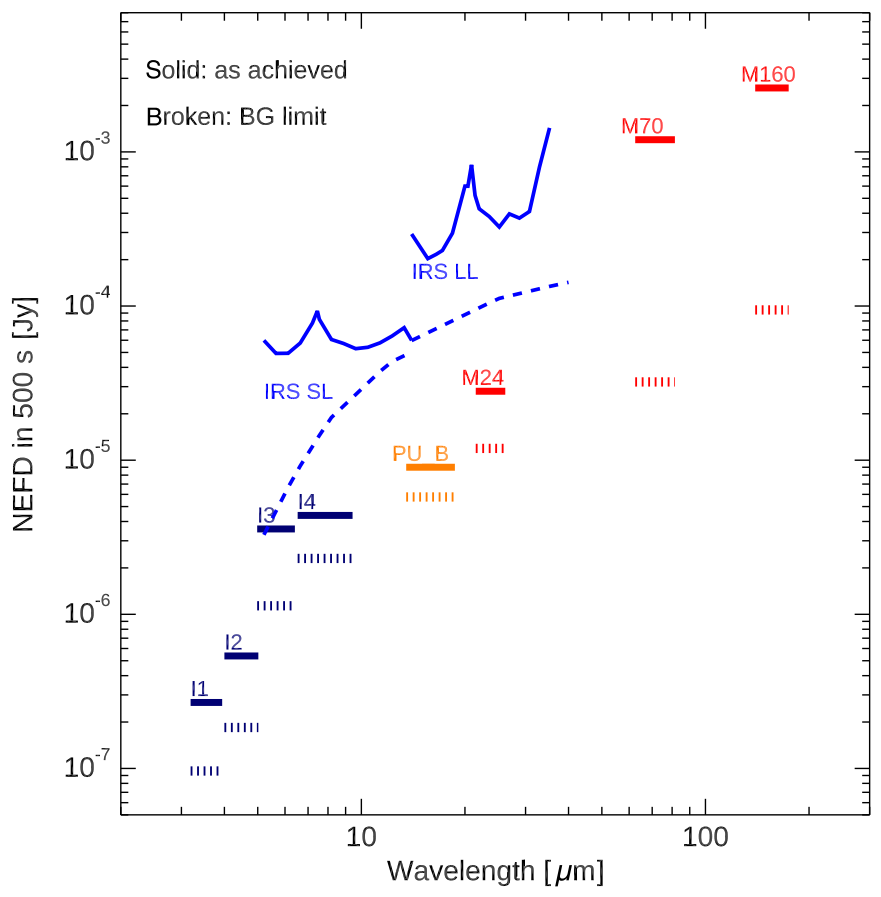

Fig. 7 Achieved Spitzer point source sensitivities (solid lines, $1 \sigma$ in $500 \mathrm{~s})^{2}$ compared with estimates of fundamental limits imposed by the zodiacal background. The short vertical lines under each instrumental bandpass show the zodiacal background-limited NEFD estimate for the bandpass, assuming noise from the zodiacal light in an annual average sightline viewing the north ecliptic pole. Note that the achieved performance is closest to the background limit in the 8 to $24 \mu \mathrm{m}$ range where the background is at is brightest. The background couples in both polarizations with a square bandpass of the plotted width and total transmission (including detector absorption efficiency) of $0.44,0.42,0.14$, and 0.30 for IRAC bands $3.6,4.5,5.8$, and $8.0 \mu \mathrm{m}$, respectively, 0.6, 0.18, and 0.15 for MIPS 24,70 , and $160 \mu \mathrm{m}$, respectively, and 0.65 for the IRS blue peak-up array (PU B) at $16 \mu \mathrm{m}$. Perfect detectors that add no noise other than generation-recombination noise are assumed throughout. The sensitivity achieved by MIPS is degraded below the zodiacal limit, in part, by the effects of confusion at both 70 and $160 \mu \mathrm{m}$; it is appreciable in both channels in $500 \mathrm{~s}$. The IRS short-low and long-low module sensitivities are referred to a $\lambda / \delta \lambda=50$ bin; the estimates adopt wavelength-independent efficiencies of $12 \%$ and $8.5 \%$, respectively; this is meant to include all sources of loss including slit coupling, blaze efficiency in both polarizations, filters, and detector quantum efficiency. For all, it is assumed that the $14 \%$ obscured $85-\mathrm{cm}$ Spitzer telescope $\left(A_{\text {geom }}=0.489 \mathrm{~m}^{2}\right)$ couples to a point source with $75 \%$ aperture efficiency. The $\sqrt{2}$ photoelectron recombination penalty is included for all bands except IRAC 1 and 2 , where it does not apply.

also assumes an ideal instrument with a large number of small noiseless pixels that can capture and properly weight the point spread function. In the case of the Spitzer instruments, the pixels are larger than this ideal (to reduce the effects of excess noise that could arise from a large number of real pixels) — which leads to excess sky background and thus to less sensitivity compared with a perfect instrument. In practice, even the zodiacal background limit will actually be degraded by galactic cirrus emission and source confusion, especially at the longer wavelengths. This is readily apparent in the figure where the measured sensitivity deviates most strongly in the MIPS long wavelength bands at 70 and $160 \mu \mathrm{m} .{ }^{17}$ We feel that Fig. 7 and the accompanying discussion show that we can build instruments fully capable of exploiting the low backgrounds of the space environment.

\subsection{Cosmic Ray Hit Rate}

Observatories operating outside the protection of the Earth's magnetosphere will be more susceptible to the effects of galactic and solar cosmic rays than their better-shielded low Earth orbit 


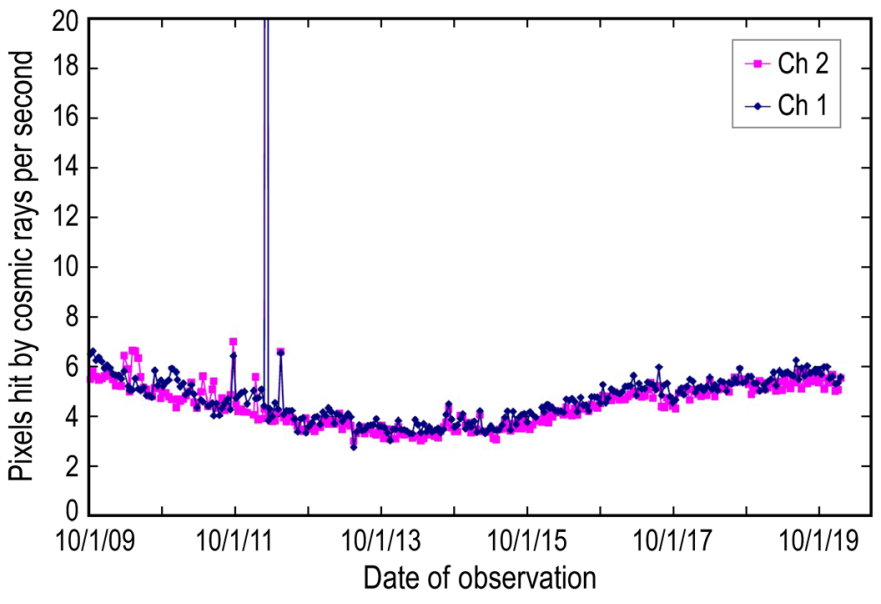

Fig. 8 Average number of pixels in the IRAC arrays affected by cosmic rays in the calibration frames taken during the Spitzer warm mission. ${ }^{3}$ The array formats were $256 \times 25630-\mu \mathrm{m}$-squared pixels. The spike in 2012 was due to a solar flare.

cousins. Spitzer was designed with radiation-hardened shielding and tested with an assumed radiation environment, and it provided data on radiation effects in the context of a modern observatory using infrared array detectors. We describe here the effects of solar and galactic cosmic rays on the pixels of the 3.6 and $4.5 \mu \mathrm{m}$ InSb arrays used by IRAC. For data processing, the number of affected pixels in a known time interval, typically $100 \mathrm{~s}$, was tracked using the dark-sky calibration observations taken with IRAC once per week and was found to correspond to 4 to 6 affected pixels per second (Fig. 8) for a $256 \times 256$ pixel array of $\sim 30 \times 30 \mu$ m pixels. The full-well capacity was $\sim 45,000 \mathrm{e}^{-}$, and the array material was InSb. The threshold for inclusion in Fig. 8 was that the pixels were 10-sigma outliers once all point sources were removed from a given frame. Multiple dithers at each pointing position allowed for the identification of cosmic ray hits even if they coincided with a point source on the sky. In most cases, only one pixel was affected at the 10-sigma level by a particular cosmic ray. The trend observed in the IRAC data followed the inverse of the solar cycle, as expected, with fewer particle hits during peak solar activity. The fact that the hit rate contained the imprint of the solar cycle shows that galactic cosmic rays, rather than solar particles, dominated the particle flux except during major solar events. These images were also used to count the number of bad, noisy, and dead pixels.

Taking 5 pixels/s as a typical hit rate, bearing in mind that each array consisted of $256 \times 256$ $30 \mu \mathrm{m}$-sized square pixels, and assuming that each incident particle liberated enough charge to register as a cosmic ray hit, the isotropic flux of ionizing particles was about $8 \mathrm{~cm}^{-2} \mathrm{~s}^{-1}$. We feel that this is broadly consistent with expectations based on the observed flux of high energy cosmic rays and energetic solar particles. It is noteworthy that the data for each of the two IRAC arrays showed the same hit rate and the same temporal behavior. This is of course what one would expect if the saturated pixels are attributable to externally incident ionizing radiation. See Ref. 18 for more information about the radiation hits and discussion of other issues related to precision warm mission photometry with Spitzer.

The IRAC data processing pipeline monitored all data, even that obtained during solar flares, to apply corrections for radiation hits before the data were released to the science investigator. Affected pixels were identified for a single observational period pointed at one part of the sky. This usually consisted of between 20 and 1000 single frames of the same exposure time. The data processing pipeline created a mask file that kept track of any problem pixels for each frame. As the telescope's field of view was dithered or scanned across the sky, an astronomical source would be observed in multiple pixels across the array, but a cosmic ray would affect only one pixel (typically) in one frame. When stacked by astronomical coordinates, an astronomical source would appear in multiple frames, so an outlier is a pixel with anomalous flux appearing in only one of the frames. Those outlier pixels were then flagged as radhits, marked in the pixel masks, and excluded when a mosaic, or image map, of the observations was created. This aided in producing artifact-clean images while preserving the processing history. Early in the mission, 
it was found that, if Spitzer were struck by a solar flare and subsequent coronal mass ejection, the number of radiation hits measured by IRAC would rise sharply and quickly fall away. This was used to determine if an observation needed to be retaken. This and other features of Spitzer's response to high energy particles, including both spacecraft and payload issues, are discussed in Ref. 19, which also discusses the effects of the space environment on the solar panel performance. In addition, the position of Spitzer in its orbit allowed data on particle hits as recorded by Spitzer to be used in partnership with data from other spacecraft throughout the inner solar system to constrain models of Solar Energetic Particle events and Coronal Mass Ejections. ${ }^{20,21}$

A figure of merit for the health of the arrays over time is the number of hot and noisy pixels. These changed with time as some pixels recovered and others became hot or noisy. Pixels were deemed noisy if the standard deviation during the IRAC measurements described above was greater than twice that of the median for surrounding pixels and hot if the pixel retained $>$ 50 counts (DN) after the flux was read out. In band 1 (the results for band 2 are similar), the number of hot pixels increased fairly uniformly from 80 to 200 over the course of the warm mission, perhaps reflecting the increased accumulated ionizing radiation dose, whereas the number of noisy pixels bounced around more randomly but generally stayed below the hot pixel count. In practice, we found that it sufficed to update the bad pixel masks about twice per year. Dead, or nonresponsive, pixels increased from about 20 to about 35 during the warm mission. The effects of cosmic ray hits were reversible, and more than $98 \%$ of pixels remained usable at end of the 16+ year mission. A hot pixel table was separately maintained for the star tracker, which used a $512 \times 512 \mathrm{CCD}$ with $20 \mu \mathrm{m}$ pixels and a "lost in space" acquisition strategy described in detail by Ref. 22. At the end of the mission, there were 46 hot pixels listed in this table.

Note that, at the Observatory level, Spitzer was protected against cosmic rays by the use of radiation hardened components, including the computer, FPGA, and optoelectronic coupler chips. It is noteworthy that, of the 19 events that caused Spitzer to enter standby or safe mode over the 16+ year mission, only one could definitely be attributed to a single event upset (SEU). This occurred in February 2009, when an SEU induced a double memory fault in the combined electronics unit that served both the IRS and the MIPS Si:As and Si:Sb arrays.

\subsection{Photometric Stability}

The IRAC team monitored the system's photometric stability at 3.6 and $4.5 \mathrm{~m}$ by periodically repeating observations of calibration stars. The calibration stars included 21 primary and secondary stars chosen at the beginning of the cryogenic mission. ${ }^{23}$ The calibrator stars were either $\mathrm{K}$ giants or A main sequence stars as it was thought that these stellar types could be well modeled and the stars were known to not have astrophysical variations at these wavelengths. Primary calibrators were located in the continuous viewing zone at the orbit poles, so an entire set of primary stars could be observed every 2 weeks. These were used to determine the flux conversion for each camera. The set of secondary calibrators had positions near the ecliptic plane, and therefore only two were visible at any given time. These were used to monitor the stability of the photometry over 12 to $24 \mathrm{~h}$ timescales.

We use seven primary calibrators to examine the measured responsivity over the final 8 years of the warm mission (Fig. 9). The calibration stars were observed with a dither pattern in full array mode. The flux densities were measured with a radius of 3 pixels and a reference sky annulus of 3 to 7 pixels. They were corrected for both the array location dependence and the pixel phase effect (see below), dithering to many positions and binning over all stars reduced systematics from each of these effects. Each individual star's measurements were normalized to the median of all observations of that star over time before being binned together with the other calibration stars at a given epoch to determine the median responsivity at that epoch. Error bars were calculated for each time-bin by taking the standard deviation of the ensemble of photometric points and dividing by the square root of the number of data points in the bin. The data at channel 1 clustered around a median flux of 1 , showing only the small variability discussed below. The channel 2 data were comparably stable but offset in the plot for clarity. All data were processed with pipeline version S19.2. For further details see Ref. 18. 


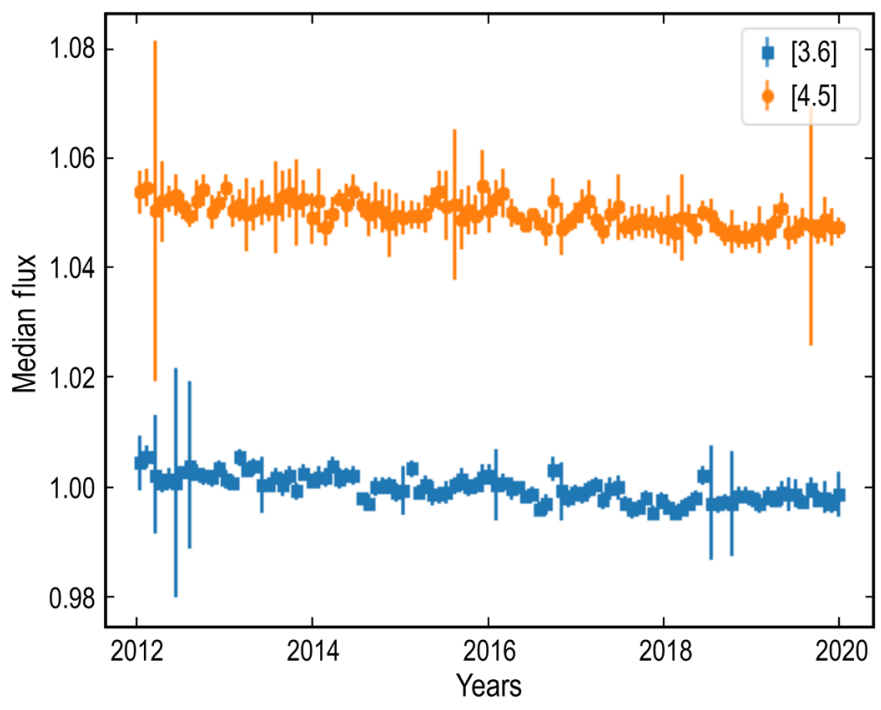

Fig. 9 Average IRAC responsivity versus time for the final 8 years of the warm mission, based on the measured flux from seven primary calibrator stars. The seven primary calibrators for Spitzer were KF09T1, KF06T2, KF08T3, KF06T1, NPM1p67, NPM1p60, and 1812095. ${ }^{23}$

Figure 9 shows a decline in responsivity of $0.1 \%$ and $0.05 \%$ per year in ch 1 and 2 , respectively, over the final 8 years of the mission. Similar studies of the cryogenic mision ${ }^{24}$ show a similar rate of drop in responsivity for the InSb arrays; unfortunately, similar data are not readily available for the other Spitzer detectors. Other than changes in dark current or noise, which were frequently reversible, there is no evidence of any degradation of the arrays with time. We feel that the most likely cause of the drop in responsivity was radiation damage to the optics, which is expected in the space environment and would cause Rayleigh scattering in the transmissive elements. Other possibilities could be radiation damage to the band-defining filters or to the beam splitters, which were used in reflection for IRAC bands 1 and 2. The materials used in these lenses, filters, and beamsplitters include $\mathrm{MgF}_{2}$ and $\mathrm{ZnS}$ lenses for the $3.6 \mu \mathrm{m}$ band and $\mathrm{ZnSe}$ and $\mathrm{BaF}_{2}$ for the $4.5 \mu \mathrm{m}$ channel. The radiation from the sky reflected off a multilayer dielectric beam splitter with a Germanium substrate, and the band-defining filters were multilayer coated Ge. Finally, the arrays were antireflection coated with $\mathrm{SiO}$. For more information, consult the IRAC instrument handbook. ${ }^{25}$

The IRAC InSb arrays were thermally stabilized at around $15 \mathrm{~K}$ during the cryogenic mission and $29 \mathrm{~K}$ for the warm mission, and the bias points were reset for optimum performance. ${ }^{6}$ As a result, the performance of IRAC was degraded by no more than $10 \%$ in going from the cryogenic to the warm mission, and the warm mission images are indistinguishable from those obtained during the cryogenic mission. Even at $29 \mathrm{~K}$, the dark current of the InSb arrays was below the photocurrent due to the zodiacal background, assuring natural-background limited performance (Fig. 7). Reference 6 discusses how several detector artifacts varied between the cryogenic and warm missions. For data on the performance of similar InSb arrays operated at higher temperatures in a similar low background environment, see Refs. 26 and 27. These papers show that more serious detector artifacts start to appear at temperatures above $40 \mathrm{~K}$.

The pixel phase effect alluded to above reflects the fact that, due to intrapixel spatial variations in effective quantum efficiency, the signal received from a star depends on the position of its photocenter on an individual 1.2" pixel. During the cryogenic mission, the pixel phase effect could cause signal variations as large as $4 \%$ (peak-to-peak) at $3.6 \mu \mathrm{m}$ but less than $1 \%$ at $4.5 \mu \mathrm{m}$. For the warm mission, the corresponding variations were $9 \%$ and $4.7 \%$, respectively. ${ }^{28}$ It can be corrected to first-order using analysis tools available at NASA's Infrared Science Archive (IRSA), which calculate the image centroid and then apply a correction, depending on the centroid position, to estimate the flux that would have been measured if the source centroid was at the position of maximum sensitivity near the center of the pixel. The correction was based on the (normalized) responsivity measured at different locations within a pixel for hundreds of pixels 
across the array. For the warm mission, the correction was a two-dimensional (2D) Gaussian centered at the peak response of the pixel (which is slightly offset from the pixel center). The same correction was used for all pixels in a given array. As will be discussed below, the pixel phase effect was particularly insidious when it was coupled with pointing drifts; even a small motion of the image within a single pixel could produce easily detectable changes in the measured flux. To be effective, both this correction and that for the array-location variation described below had to be applied to individual data frames prior to any mosaicking or co-adding.

The array-location-dependent correction was required to compensate for the fact that IRAC was flat-fielded using the zodiacal background, which was not valid for compact sources with star-like spectral energy distributions. It also responded to the fact that, as expected, the filter effective wavelength varied across the array due to the change in incidence angle of the radiation from the sky. This effect could change the inferred flux by $1.3 \%$ on average, with the effect increasing to $\sim 5 \%$ for stars at the edge of the array in IRAC1 and $8 \%$ in IRAC2. Again, tools made available by the Spitzer Science Center and available at IRSA correct this effect on a perpixel basis using a 2D numeric array, which also corrects for the small effects of the spatial distortions across the detector array.

\section{Spacecraft Performance}

\subsection{Electrical Power Generation}

A degradation in material properties similar to that which led to the increased solar panel temperature shown in Fig. 4 above led to a decrease in the power provided by the solar panels over time. Figure 10 shows the average current produced by Spitzer's solar panels over the mission. A gradual decrease in output current is seen with the familiar annual modulation due to the orbital eccentricity. Also shown is a sharp drop in output in November, 2010, which is inferred to be a micrometeoroid impact that disabled a portion of one of the strings of solar cells in one of the panels. Even with that loss of capability, however, the average power generated by the solar panels was adequate to meet the needs of the observatory with margin.

\subsection{Pointing System Performance}

Spitzer incorporated a sophisticated realization of a standard pointing system with gyros and a star tracker to establish and maintain the orientation of the spacecraft and a sensor within the focal plane that monitored the coalignment of the telescope line of sight with that of the spacecraft. ${ }^{29}$ Slews for target acquisition or within an observing sequence were carried out

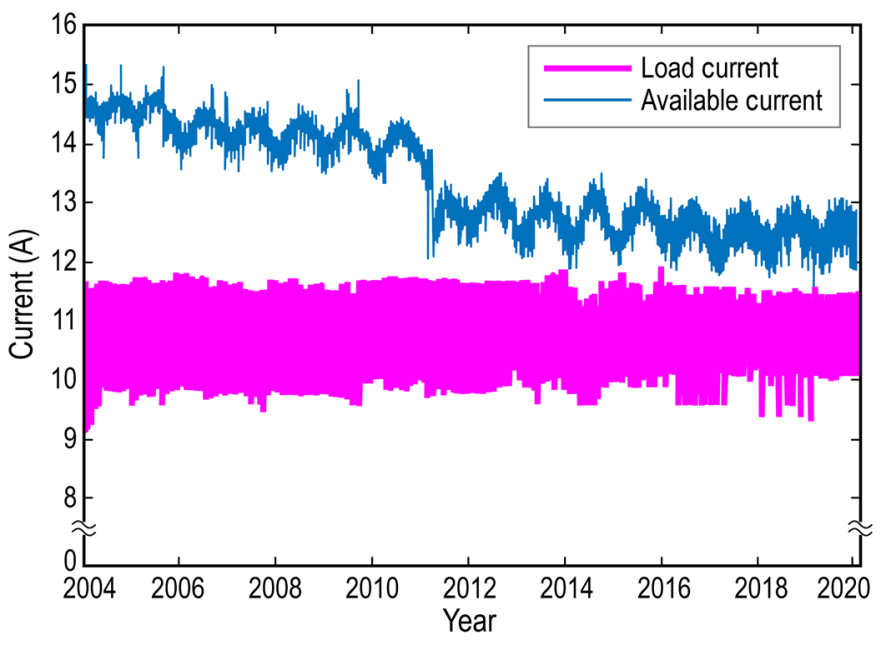

Fig. 10 Average solar panel-generated current over time for the entire Spitzer mission. 
by a set of four reaction wheels. Thermal considerations required that the telescope was only weakly connected, mechanically, to the spacecraft, Pointing offsets and drifts between the telescope and star tracker lines of sight were monitored and corrected with the help of the PCRS, a pair of $4 \times 4$ arrays of Si p-i-n diodes that were installed in the cold focal plane and provided visible light images of stars also seen by the spacecraft star trackers. Spitzer's star-tracker-totelescope alignment calibration was performed by taking the centroid of a single star in the PCRS sensor once every $8 \mathrm{~h}$. Each single-star calibration update was processed using a Kalman filter denoted as the S2P (Star-tracker-to-PCRS) calibration filter. ${ }^{30}$ S2P alignment was accurate to $\sim 0.44$ " just prior to the S2P update and to 0.35 " just after the S2P update (both $1-\sigma$, radial). This 0.35 " to 0.44 " accuracy was maintained throughout the mission. Without the S2P calibration, this critical alignment could drift by as much as 1 or $2 \operatorname{arcsec}$ in 10 days. The placement of targets on the desired pixel or spectrograph slit was facilitated as well by a precise survey of the Spitzer focal plane carried out during IOC. ${ }^{31}$

The offsets and drifts were kept minimal by the fact that the telescope and the spacecraft were shaded from sunlight - and thus kept in a stable thermal configuration - by the solar panel and its extension, In addition, a heat pipe was used to isothermalize the spacecraft top deck, just below the attachment points of the telescope struts, to minimize the effect of changes in the thermal state of the spacecraft on the telescope line of sight. The performance and reproducibility of the system was further improved by the fact that the two instruments for which system target acquistion was most critical, the IRAC and the IRS, had no moving parts.

During the cryogenic mission, the most critical absolute pointing requirements were established by the Infrared Spectrograph (IRS), which required that targets be placed on the spectrograph slits with sub-arcsecond precision. This was achieved by the use of "peak-up arrays" within the IRS that provided infrared images of the target so that the telescope could be offset to bring the target from the peak-up array on to the spectrograph slit. As many of the science targets were detectable only in the infrared, the visible light star trackers and PCRS were not useful for this purpose. This system performed very well for most purposes, achieving pointing and offset accuracies better than 0.5 " ( $1-\sigma$ rms radial $)$, with jitter less than 0.03 " $(1-\sigma \mathrm{rms}$ radial) over $600 \mathrm{s.}^{29} \mathrm{Here}$, we focus on adaptations to this basic system that were driven by the use of Spitzer to observe exoplanets, or planets around stars other than the Sun, which became a major science theme for Spitzer within a few years of launch. Exoplanet studies required nonstandard approaches to both the understanding and the use of the pointing and control system.

The discovery of the first transiting exoplanets in 2002 led to the realization that Spitzer was capable of measuring not only the depth of a transit, which occurs when the planet moves in front of the star and blocks its light temporarily, but also the depth of a secondary eclipse, which occurs when the planet moves behind the star and its infrared light is no longer seen. Spitzer observations of secondary eclipses of two exoplanets, published in $2004,{ }^{32,33}$ were the first detections of light from exoplanets. In subsequent years, exoplanet studies became an important and growing portion of the Spitzer science program. Exoplanet studies required measurement precision better than 100 parts per million over periods of hours, far in excess of anything that had been achieved at Spitzer's wavelengths from previous ground- or space-based telescopes, including Spitzer. Spitzer users and project personnel worked together over a period of several years to understand how to use both the instruments and the spacecraft, including the pointing system, in previously unanticipated ways to optimize the collection of data on exoplanets and how to analyze the data to achieve the required levels of precision.

The depth of an exoplanet transit was less than $1 \%$ and often less than $0.1 \%$ of the signal, so it was crucial to correct for intrapixel variations to obtain any science. Some of the first exoplanet observations employed a dithering strategy (intermittently shifting the position of a target on an array); however, even after the dithering strategy was abandoned in favor of keeping the target fixed on a well-characterized pixel, the main systematic noise limiting the precision of photometry was found to be small changes in the pointing position coupled with the intrapixel gain variations. Attempts to understand and mitigate these and other known systematics have been accomplished through the following operational changes: increasing target acquisition precision and repeatability, independently calibrating the intrapixel gain map, and increasing the overall stability of pointing. The application of these techniques was validated by several communitywide data challenges. ${ }^{34}$ 


\subsubsection{Target Acquisition}

The intrapixel gain variations could exceed $4 \%$ in band 2 , depending on where in the $1.2 \times 1.2$ arcsec pixel the target centroid fell; they were larger in band 1 . To mitigate this, a single pixel (the "sweet-spot" pixel) at the center of each of the IRAC subarray sections (a $32 \times 32$ pixel unit within the larger $256 \times 256$ array that was used to achieve higher sampling frequency and make observable brighter targets than could be measured using the entire array) was selected. The PCRS was used to enhance the repeatability of target acquisition by placing the target star on the sweet-spot pixel. The PCRS operated in the visual part of the spectrum (505 to $595 \mathrm{~nm}$ ), and its main function was to calibrate and remove the optical offset between the star trackers and the telescope. In effect it was "repurposed" to provide a means of placing optically bright exoplanet host stars on the sweet-spot described below.

The PCRS could measure the centroids of stars in the $7.0 \mathrm{mag}<V<11.8 \mathrm{mag}$ range to within 0.14 " $(1-\sigma$ radial $)$ and feed these data, in the form of a pointing correction, to the spacecraft pointing control system. The PCRS peak-up repeatedly placed a target within an $0.5 \times 0.5$ pixel sweet-spot on the designated 1.2" pixel (see Fig. 11). The highest precision was obtained when the peak-up star was also the planet-bearing star that was the target of the observation. For fainter host stars, the peak-up was done on a brighter nearby star, and the system performance degraded slightly. When the peak-up target was the science target, more than $90 \%$ of visits were placed somewhere inside the sweet-spot; $68 \%$ of visits were within 0.1 pixels of the center of the sweet-spot; and repeat visits to the same target resulted in a spread of initial positions less than 0.06 pixels $68 \%$ of the time. This gave two advantages: (1) it minimized the offset in intrapixel gain caused by initial target acquisition, and (2) it allowed us to map out the responsivity of a small portion of the designated pixel with high fidelity to facilitate data analysis.

For long exoplanet observations, particularly during the latter phases of the warm mission, Spitzer was susceptible to long-term pointing drifts following downlinks, as the system relaxed thermally after the heating of the spacecraft during the off-sun excursions. Coupled to the intrapixel gain variations, these could become problematic when lengthy exoplanet observations, which could extend over $24 \mathrm{~h}$ or more, were executed. Investigation of the pointing of Spitzer on a range of time scales showed that on top of the thermal relaxation there was a roughly constant, long-term drift of 0.34 arcsec per day. ${ }^{35}$ This drift was due to an inconsistency in the way that velocity aberration corrections were handled by the spacecraft's Command and Data Handling computer $(\mathrm{C} \& \mathrm{DH})$ and by the star trackers. Whereas the $\mathrm{C} \& \mathrm{DH}$ assumed that velocity aberration would be constant over the course of each observation, the star trackers updated the velocity aberration corrections continuously. The result was a constant error signal sent by the star trackers, which the spacecraft constantly attempted to correct, pulling the line of sight away from the initial pointing position. The strategy adopted to deal with the pointing drifts was twofold. The first was a $30 \mathrm{~min}$ "cooling off period" following a return to the observing orientation before any pointing-critical observations were carried out. During this time period, the coalignment of the telescope and the spacecraft relaxed to the usual subarcsecond level. This was augmented by repeated executions of the peak-up procedure, carried out about every $12 \mathrm{~h}$, which led to a repointing and dealt with all long-term drifts.

\subsubsection{Mapping the sweet-spot}

The availability of the PCRS peak-up capability facilitated detailed mapping of the gain variation around the sweet-spot. In a typical 12-h time series measurement, the coupling of pointing fluctuations and intrapixel gain variations could cause measured fluxes to vary by more than several percent. Usually, these variations were removed from the science data (selfcalibration) by decorrelating the systematics from the signal using quantities, such as $x$ and $y$ centroids, that changed in tandem with pointing fluctuations and thus were independent of astrophysical variations. However, the aliasing of astrophysical and instrumental variations was always a possibility and could not be ignored without an accurate model of the variations of either the astronomical system under study (which would limit observers to previously studied systems) or the instrumental gain. Thus, to permit an independent estimate of the position-dependent instrumental gain,

J. Astron. Telesc. Instrum. Syst. 014002-19 Jan-Mar 2022 • Vol. 8(1) 
the IRAC instrument science team accumulated a set of $\sim 1$ million repeated photometric observations of nonvariable calibration stars in channels 1 and 2 over the sweet-spot region of each array. The data set required about $150 \mathrm{~h}$ of observatory time. The responsivity maps ${ }^{28}$ show that, even over the $0.5 \times 0.5$ pixel central region of the sweet-spot, the gain in IRAC band 1 varies $3 \%$ to $5 \%$. The use of the sweet-spot gain map in analyzing exoplanet time series data was one of the data reduction schemes compared in Ref. 34.

\subsubsection{Improving pointing stability}

Soon after scientists started systematic exoplanet studies with Spitzer, it became apparent that there was a persistent modulation of the signal seen by IRAC with a period of about $1 \mathrm{~h}$ and a peak-to-peak amplitude of $0.4 \%$ (Fig. 12). This could readily be seen in the time series photometry of measurements of bright stars. This 1-h period was inconveniently close to the length of many of the exoplanet transit and eclipses being studied by Spitzer users, and the amplitude of the modulation was large compared with the precision required for many Spitzer exoplanet observations. A search of spacecraft engineering records revealed that a battery heater was cycling on and off with just this period, which was established in large part by the dead band over which the temperature was allowed to drift before the heater state changed autonomously. It was suspected that the change in heater power changed the thermal state of the spacecraft, introducing a slight offset between the star tracker and telescope lines of sight. Because the pointing position was established by star tracker observations, this, in turn, led to a change in the observed signal due to uncorrected intrapixel gain variations. The $0.4 \%$ signal modulation initially reported, corresponding to a 0.1 pixel peak-to-peak pointing oscillation, was large compared with the $0.01 \%$ (100 parts per million) precision required for many of Spitzer's exoplanet studies. In response, the Spitzer engineering team tightened the heater deadband so that it cycled on a period of $\sim 40$ min leading to a pointing fluctuation of $\sim 0.05$ pixel peak-to-peak and a signal modulation of $0.2 \%$ (Fig. 12). Separating the instrumental and exoplanetary timescales and reducing the amplitude of the pointing fluctuations led to increased photometric precision by facilitating more precise subtraction of this signal variability as part of the data analysis.

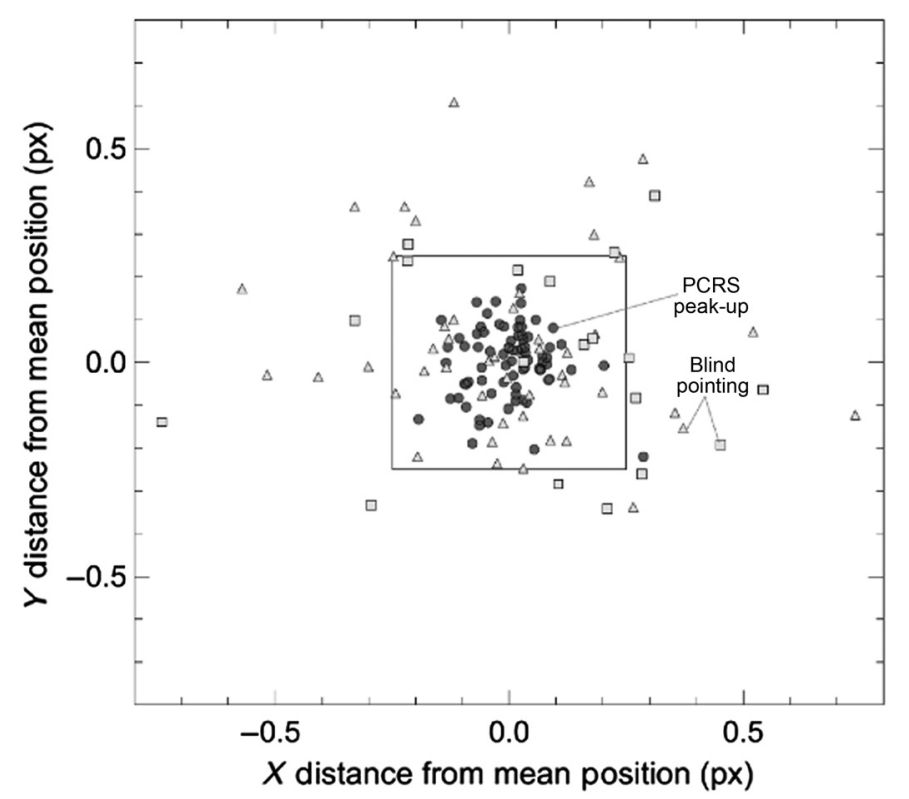

Fig. 11 Comparison of pointing repeatability using peak-up versus blind pointing. Each point represents the mean centroid of the first 64 frames of the following: 78 observations (dark circles) beginning with PCRS peak-up followed by an offset to the $4.5-\mu \mathrm{m}$ sweet-spot using gyros; 20 observations (light squares) using PCRS peak-up and the star tracker for the offset; and 48 observations (light triangles) initiated without PCRS peak-up. Each set of symbols has had the mean position of the set subtracted. A $0.5 \times 0.5$ pixel square is overlaid on the plot, indicating the size of the sweet-spot region. ${ }^{28}$ 
The close partnership between the Spitzer users and the Spitzer Science Center facilitated the reporting and tracking down of this troublesome effect, as it was much more apparent in the science data than in the engineering data from the pointing system.

\subsection{Angular Momentum Management}

Prior to launch, it was recognized that the pressurized nitrogen gas that was used with Spitzer's cold gas thrusters to unload angular momentum accumulating in the reaction wheels was a potential life-limiting consumable. The center of pressure vector from the solar radiation impinging on the observatory did not pass through the center of mass, so solar radiation pressure produced a net torque around the center of mass. As the helium cryostat was located close to the observatory center of mass, boil-off of the He liquid had minimal effect on this offset over the course of the mission. (The difference in the location in the observatory center of mass between a full and an empty cryostat was $<5 \mathrm{~cm}$.) The torque due to the radiation pressure had to be counteracted with the reaction wheels. If the observatory spent too long in one orientation, this momentum built up in the wheels and would have to be unloaded using the cold gas thrusters, expending some of the onboard nitrogen gas each time. (By contrast, in low Earth orbit this can be accomplished by magnetically torquing against the Earth's magnetic field.) Note that the thrusters were not intended to provide a delta- $\mathrm{v}$ to the observatory, only to provide a rotational torque to unload the reaction wheels.

If observations were planned so that equal time was spent looking down as looking up with respect to the orbital plane, the momentum buildup in the wheels due to the solar radiation pressure in one orientation could be canceled. As it turned out, this was not a major driver in planning observing sequences: optimizing the time spent on observations of the science targets was more important. Of greater consequence was the fact that, as with time the momentum buildup was understood, it became possible to schedule unloading of the stored angular momentum at a benign time to avoid disturbing the spacecraft with an autonomous desaturation at a critical time,

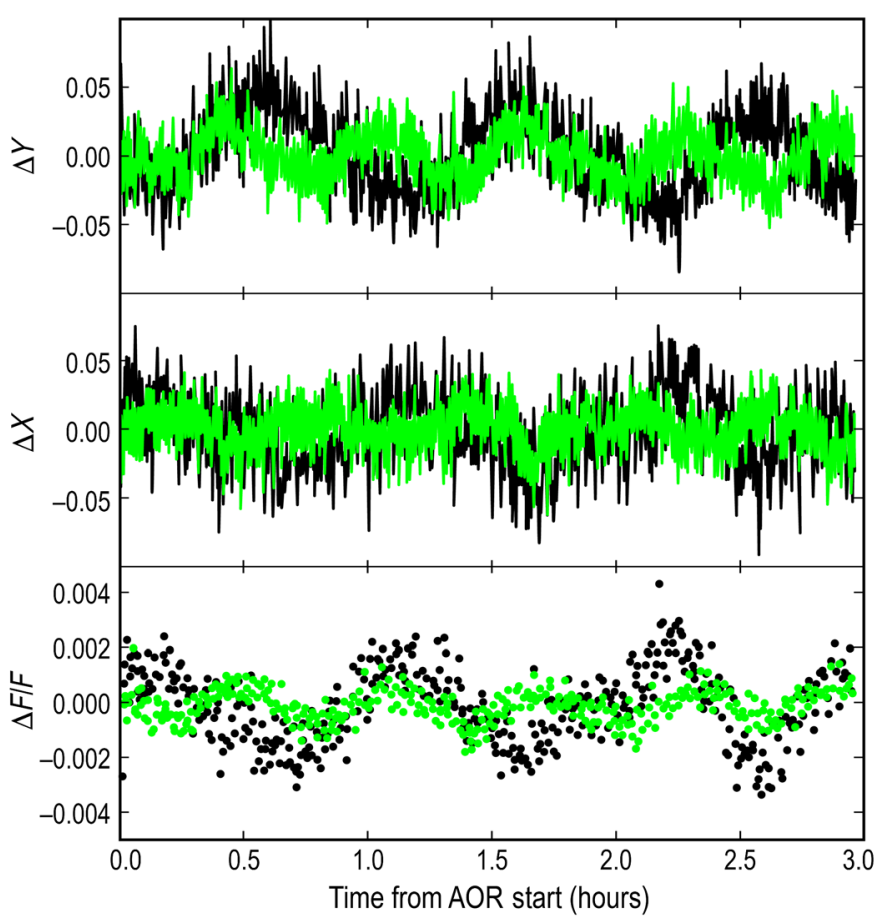

Fig. 12 Effects of tightening battery heater dead band on pointing and photometric stability. The upper two panels show the variation of the $(x, y)$ position of the centroided stellar image on the array, and the lower panel shows the variation in measured signal, all as a function of time. Black points are data taken before the dead band was tightened; green points were taken after the adjustment. 
such as during a long integration on a target. At the end of the mission in January $2020,<60 \%$ of the $15.6 \mathrm{~kg}$ of $\mathrm{N}_{2}$ carried into orbit had been used. Thus, there was some conservatism in the reaction control system design, which was appropriate since the rate of angular momentum buildup would depend on the exact observing scenario. There might have been some science observations proposed that would have led to a greater imbalance in the observing time spent looking "up" versus "down." As it was, there were 1066 desats (with apologies to William the Conqueror) during the 2030 day Spitzer mission. Thus, on average it took about 2 days for the angular momentum to build up in the reaction wheels to the point where it tripped preset limits for desaturation or a desaturation was commanded in advance of a long and critical observation.

\section{Lessons Learned and Conclusions}

In 2010, coauthors Werner and Roellig collaborated with Robert Gehrz on an SPIE publication discussing lessons learned from Spitzer. ${ }^{12}$ These included scientific, technical, and management issues but were necessarily weighted toward the cryogenic mission. We describe below several additional lessons that have risen to the top as we look at Spitzer through the lens of the warm mission.

\subsection{Radiative Cooling}

Spitzer's innovative use of radiative cooling has been particularly significant. From the point of view of evaluating the durability of the radiative cooling alone, we can say that, after 16+ years at 1 AU from the sun, a well-designed and well-constructed radiatively cooled system maintained the telescope temperature at $26 \mathrm{~K}$ to within a fraction of a degree. Stepping outward, the temperature of the outer shell increased by no more than $1.5 \mathrm{~K}$ over the $16+$ years, and much or all of that can be attributed to the increased power absorbed by the solar array, which trickled down to the outer shell. So there is no evidence over this time period of degradation in the low temperature thermal and optical properties of the components of the system responsible for the radiative cooling. It is also noteworthy that the cryogenic lifetime of Spitzer was predicted to be better than $5 \%$ from prelaunch models and the results of thermal balance tests on the ground. In Sec. 2.6, we list several other "lessons learned" relevant to the cryothermal system. In closing, we emphasize once again that the success of the Spitzer cryothermal system is a tribute not only to the quality of the design but also to the skill and care with which the system was assembled and tested by the Ball team.

\subsection{Extended Mission}

Several of our thoughtful referees' reports asked what had been done during the cryogenic mission, which was the "prime mission" for Spitzer, to prepare or optimize for the warm mission, which in NASA jargon is an "extended mission." Our answer was simple: nothing. We feel that it would be a bad idea to get distracted during the development of a system for its prime mission to focus any resources or energy on considerations of a possible extended mission. The best guarantee of a successful extended mission will be a successful prime mission.

\subsection{Teaming Is Important}

There are two sides to this. Spitzer benefited immeasurably from good relationships with our major industrial contractors, Ball Aerospace and Lockheed Martin. Spitzer management took every possible step to integrate these contractors into all appropriate activities while empowering them to use their own processes and methodologies. Spitzer scientists visited the contractors periodically over the years to discuss Spitzer science and build additional bridges to the contractors. As a result, the contractors were fully engaged during the mission, and, in particular, we had excellent support from the Observatory Engineering Team at Lockheed Martin during the warm mission when it became necessary to use the spacecraft in previously unanticipated ways. At the other end of the chain, we found that close relationships with our user community were

J. Astron. Telesc. Instrum. Syst. $\quad$ 014002-22 Jan-Mar 2022 • Vol. 8(1) 
important in maximizing the scientific output of the observatory. Perhaps the best example of this comes from the exoplanet investigations, in which issues with the pointing and control system at a scale finer than the PCS sensors could detect were identified by Spitzer's users in the scientific data and brought to the attention of the observatory staff. Future missions should remain alert to the possibility that important engineering information may be gleaned more easily from the scientific data than from the spacecraft telemetry.

\subsection{Be Alert to the Possibility of Repurposing Existing Flight Hardware}

The best example of this is the use of the PCRS for precise target acquisition and positioning during exoplanet phase curve measurements. This was not envisioned when the PCRS was designed to track pointing offsets between the telescope and the spacecraft. A slightly quirky example of this was the use of data from Spitzer to set limits on the characteristics of Coronal Mass Ejections, which was made possible by its unique position in the solar system.

We hope that this distillation of the Spitzer experience demonstrates the remarkable engineering performance of the Spitzer Space Telescope and that it will be of use to colleagues working on future astrophysical observatories in space. We have limited the discussion to areas in which the Spitzer experience is novel and/or likely to be of general interest. In doing so, we have passed over a number of topics in which Spitzer's performance has been noteworthy and that may contain lessons for future missions. These include operational innovations that are not touched upon in this paper. Many of these other topics are described in a series of papers listed in the Appendix.

\section{Appendix A: Bibliography of Spitzer Resources with an Emphasis on Mission Science Operations}

\subsection{General Archives}

\subsubsection{Spitzer documentation and tools}

Spitzer technical information, including instrument and telescope handbooks, etc.: Ref. 36.

\subsubsection{Spitzer's scientific data}

Spitzer Heritage Archive: Ref. 37.

\subsubsection{Overview of Spitzer science}

References 38 and 39.

\subsection{Papers of General Interest}

Refs. 3, 12.

\subsection{Papers Related to Mission Design and Mission Operations}

References 40-49.

\subsection{Papers Related to Science Operations}

References 50-52.

\subsection{Papers Describing In-Orbit Checkout}

References 53 and 54. 


\section{Acknowledgments}

We thank JATIS editor Mark Clampin for suggesting that we include a discussion of integration and test in the paper. We thank Sean Carey of the Spitzer Science Center for providing Fig. 12. We also thank Tom Soifer of the Spitzer Science Center for his helpful comments on the manuscript and Wayne Evenson of Lockheed Martin, Denver, for early versions of Figs. 3-5. Our colleagues Paul Chodas, Bob Gehrz, Erick Young, and Dave Bayard cheerfully provided answers to numerous questions about Spitzer. Insightful comments of three referees led to significant improvements in this paper. The research was carried out at the Jet Propulsion Laboratory, California Institute of Technology, under a contract with the National Aeronautics and Space Administration (80NM0018D0004). This work is based in part on observations made with the Spitzer Space Telescope, which was operated by the Jet Propulsion Laboratory, California Institute of Technology, under a contract with NASA.

\section{References}

1. R. D. Gehrz et al., "The NASA Spitzer Space Telescope," Rev. Sci. Instrum. 78, 011302 (2007).

2. M. W. Werner et al., "The Spitzer Space Telescope mission," Astrophys. J. Suppl. Ser. 154, 1-9 (2004).

3. P. Lowrance et al., "Spitzer Space Telescope: innovations and optimizations in the extended mission era," in Space Operations: Inspiring Humankind's Future, H. Pasquier et al., Eds., SpaceOps Conference Series (2019).

4. P. T. Finley, "Spitzer cryogenic telescope assembly performance update," Proc. SPIE $\mathbf{5 8 8 3 ,}$ 12-18 (2005).

5. R. Franck et al., "High performance cryogenic radiators for James Webb space telescope," in 46th Int. Conf. Environ. Syst. (ICES) Conf. Ser. ICES-2016-141 (2016).

6. S. J. Carey et al., "Calibration and data quality of warm IRAC," Proc. SPIE 7731, 77310N (2010).

7. C. R. Lawrence and P. T. Finley, "Optimizing cryogen utilization on Spitzer Space Telescope," Proc. SPIE 5487, 124-133 (2004).

8. M. Werner, "The Spitzer Space Telescope," Opt. Eng. 51, 011008 (2012).

9. P. T. Finley, R. L. Oonk, and R. B. Schweickart, "Thermal performance verification of the SIRTF cryogenic telescope assembly," Proc. SPIE 4850, 72-82 (2003).

10. P. T. Finley, R. B. Schweickart, and R. A. Hopkins, "The space infrared telescope facility (SIRTF) cryogenic telescope assembly (CTA) cryogenic and thermal system," Cryogenics 44, 367-373 (2004).

11. R. A. Hopkins et al., "Cryogenic/thermal system for the SIRTF cryogenic telescope assembly," Proc. SPIE 4850, 42-49 (2003).

12. R. D. Gehrz, T. L. Roellig, and M. W. Werner, "Writing a success story: lessons learned from the Spitzer Space Telescope," Proc. SPIE 7796, 779602 (2010).

13. W. F. Hoffmann et al., "Simfit and focus diversity: methods for determining the focus of the SIRTF telescope in space without a focus slew," Proc. SPIE 4850, 428-440 (2003).

14. R. D. Gehrz and E. A. Romana, "Charter and activities of the SIRTF focus in-orbit checkout (IOC) integrated products team (IPT) and Optical Performance of the CTA," Proc. SPIE 4850, 62-71 (2003).

15. G. G. Fazio et al., "The infrared array camera (IRAC) for the Spitzer Space Telescope," Astrophys. J. Suppl. 154, 10-17 (2004).

16. "IRAC Image Quality," Spitzer Documentation and Tools, September 2021, https://irsa.ipac .caltech.edu/data/SPITZER/docs/irac/iracinstrumenthandbook/5/

17. H. Dole et al., "Confusion of extragalactic sources in the mid- and far-infrared: Spitzer and beyond," Astrophys. J. Suppl. Ser. 154, 93-96 (2004).

18. P. J. Lowrance et al., "Calibration trending in the Spitzer beyond era," Proc. SPIE 10704, 1070427 (2018).

19. L. Y. Cheng et al., "Investigating space weather events impacting the Spitzer Space Telescope," in Space Oper.: Innov. Invent. and Discov. SpaceOps Conf. Ser. (2015). 
20. E. Palmerio et al., "CME magnetic structure and IMF preconditioning affecting SEP transport," Space Weather 19(4), e2020SW002654 (2021).

21. T. Amerstorfer et al., "Ensemble prediction of a halo coronal mass ejection using heliospheric imagers," Space Weather 16(7), 784-801 (2018).

22. R. W. H. van Bezooijen, "SIRTF autonomous star tracker," Proc. SPIE 4850, 108-121 (2003).

23. W. T. Reach et al., "Absolute calibration of the infrared array camera on the Spitzer Space Telescope," Proc. Astron. Soc. Pac. 117, 978-990 (2005).

24. J. E. Krick et al., "Spitzer IRAC sparsely sampled phase curve of the exoplanet Wasp-14B," Astrophys. J. 824, 27 (2016).

25. "IRAC," Spitzer Documentation and Tools, September 2021, https://irsa.ipac.caltech.edu/ data/SPITZER/docs/irac/

26. S. Baba et al., "Revised wavelength and spectral response calibrations for AKARI nearinfrared grism spectroscopy: post-cryogenic phase," Publ. Astron. Soc. Jpn. 71, 2 (2019).

27. T. Mori et al., "Laboratory and in-flight measurements at 10-55 K of InSb near-infrared detector performance for the warm mission of the AKARI satellite," Publ. Astron. Soc. Pac. 123, 942 (2011).

28. J. G. Ingalls et al., "Intra-pixel gain variations and high-precision photometry with the Infrared Array Camera (IRAC)," Proc. SPIE 8442, 84421Y (2012).

29. D. S. Bayard, "Advances in precision pointing control for NASA's Spitzer Space Telescope," in Advances in the Astronautical Sciences Series, J. D. Chapel and R. D. Culp, Eds., Vol. 118, pp. 04-071, American Astronautical Society (2004).

30. D. S. Bayard, "Estimation filter for alignment of the Spitzer Space Telescope," in NASA Tech Briefs, NPO-73375 (2007).

31. D. S. Bayard et al., "Focal plane calibration of the Spitzer Space Telescope," IEEE Control Syst. Mag. 29, 47-70 (2009).

32. D. Deming et al., "Infrared radiation from an extrasolar planet," Nature 434, 740-743 (2005).

33. D. Charbonneau et al., "Detection of thermal emission from an extrasolar planet," Astrophys. J. 626, 523-529 (2005).

34. J. G. Ingalls et al., "Repeatability and accuracy of exoplanet eclipse depths measured with post-cryogenic Spitzer," Astron. J. 152, 44 (2016).

35. C. J. Grillmair et al., "Improving our understanding of the Spitzer Space Telescope's pointing drifts," Proc. SPIE 9143, 914359 (2014).

36. Spitzer Documentation and Tools, September 2021, https://irsa.ipac.caltech.edu/data/ SPITZER/docs/.

37. Spitzer Heritage Archive, Infrared Science Archive, https://irsa.ipac.caltech.edu/Missions/ spitzer.html.

38. "A Spitzer retrospective," Nat. Astron., 4, 293 (2020).

39. M. Werner and P. Eisenhardt, More Things in the Heavens, Princeton University Press (2019).

40. S. J. Barba et al., "Planning and scheduling the Spitzer Space Telescope," Proc. SPIE, 6270, 62700Z (2006).

41. J. C. Hunt and Y. C. Leo, "Re-engineering the mission operation system (mos) for the prime and extended mission," in 12th Int. Conf. Space Oper. (AIAA SpaceOps 2012), p. 1285801 (2012).

42. J. H. Kwok et al., "Spitzer Space Telescope mission design," Proc. SPIE 5487, 201-210 (2004).

43. W. A. Mahoney et al., "Spitzer scheduling challenges: cold and warm," Proc. SPIE 7016, 70161W (2008)

44. W. A. Mahoney et al., "Spitzer warm mission transition and operations," Proc. SPIE 7737, $77371 \mathrm{~W}(2010)$.

45. W. A. Mahoney et al., "Spitzer operations: scheduling the out years," Proc. SPIE 8448, $84481 Z$ (2012).

46. M. A. Sarrel and J. C. Hunt Jr., "Evaluating requirements on the Spitzer mission operations system based on flight operations experience," Proc. SPIE 7016, 70161P (2008). 
47. E. Scire, "Warm Spitzer: effects of major operational changes on publication rates," in Astron. Data Anal. Software and Syst. XXIII, Vol. 485, p. 481 (2014).

48. C. P. Scott, B. E. Kahr, and M. A. Sarrel, "Spitzer observatory operations: increasing efficiency in mission operations," Proc. SPIE 6270, 62701B (2006).

49. C. P. Scott and R. K. Wilson, "Spitzer pre-launch mission operations system: the road to launch," Proc. SPIE 6270, 627013 (2006).

50. S. R. Dodd, "The Spitzer science operations system: how well are we really doing?" Proc. SPIE 5487, 158-165 (2004).

51. L. J. Storrie-Lombardi, "Spitzer Space Telescope: unprecedented efficiency and excellent science on a limited budget," in Astron. Data Anal. Software and Syst. XXI, Astron. Soc. Pac. Conf. Ser., No. 461, Astronomical Society of the Pacific, San Francisco, California, pp. 125-134 (2012).

52. L. J. Storrie-Lombardi et al., "Lessons learned in extended-extended Spitzer Space Telescope operations," Proc. SPIE 10704, 107041D (2018).

53. S. H. Linick et al., "Spitzer Space Telescope in-orbit checkout and science verification operations," in AIAA SpaceOps 2004 Conf. (2004).

54. J. W. Miles et al., "Execution of the Spitzer in-orbit checkout and science verification plan," Proc. SPIE 5487, 134-145 (2004).

Michael W. Werner is the project scientist of the Spitzer Space Telescope at the Jet Propulsion Laboratory, California Institute of Technology. He has also served as the chief scientist for Astronomy and Physics at JPL. He has authored and coauthored dozens of papers presenting scientific results from Spitzer and is the author, with coauthor Peter Eisenhardt, of the book More Things in the Heavens: How Infrared Astronomy is Expanding our View of the Universe, which discusses Spitzer science in depth.

Patrick J. Lowrance is a senior staff scientist at Caltech/IPAC specializing in brown dwarfs and exoplanet science. He is currently part of the ground system team of the Roman Space Telescope Science Support Center. He has worked with different aspects of the science and operations of space-based observatories and instruments including the Hubble and Spitzer Space Telescopes for over two decades.

Tom Roellig has worked in the NASA civil service as an astrophysicist at the NASA Ames Research Center since 1980 and is currently the chief of the Astrophysics Branch at Ames. His scientific research interests have spanned a wide range of infrared astronomy and astronomical instrumentation development. He has conducted research and published papers in infrared instrument development, solar science, solar system science, star formation, interstellar medium, and brown dwarf astronomy.

Varoujan Gorjian is a research scientist at NASA's Jet Propulsion Laboratory, California Institute of Technology. He has been involved with the Spitzer Space Telescope for over 20 years as both a member of the Spitzer Project Science office at JPL as well as a scientific user of the telescope. His main astronomical interests are variability in active galactic nuclei, characterization of exoplanets, and detection of the cosmic infrared background.

Joseph Hunt, Jr., is a project manager at NASA's Jet Propulsion Laboratory in Pasadena, California, USA. One of his most passionate past roles was deputy mission manager and flight director for NASA's Spitzer Space Telescope mission. He has over 42 years of diverse aerospace engineering experience with professional expertise in the disciplines of flight simulation, inertial measurement units, Earth orbiting satellites, and interplanetary and deep space spacecraft, flight, ground and mission operations systems and processes. He currently serves as the project manager for the Mars Odyssey and NEOWISE missions.

Charles M. Bradford obtained his doctorate in astronomy and instrumentation at Cornell in 2001. He held a Millikan postdoctoral fellowship at Caltech from 2001 to 2003 and has been on the science staff at JPL since that time. He enjoys developing and fielding new submillimeter and millimeter-wave instrumentation on mountain-top sites, using the datasets to study interstellar 
medium conditions in galaxies near and far. Current projects include a balloon-born far-IR spectrometer, an on-chip mm-wave spectrometer demonstration, and a mm-wave line intensity mapping instrument. He is also active in developing concepts and the necessary detector systems for cryogenic space far-IR astrophysics missions.

Jessica Krick is an astronomer and data scientist on the Infrared Science Archive science platform team at Caltech/IPAC. She received her $\mathrm{PhD}$ in astronomy and astrophysics from the University of Michigan and has worked as part of the Spitzer IRAC instrument support team. Her scientific interests range from zodiacal light to exoplanets to clusters of galaxies and include machine learning and data science techniques applied to big astronomical datasets. 